Historic, archived document

Do not assume content reflects current scientific knowledge, policies, or practices. 



\section{CASE STUDY: GROWTH AND DEVELOPMENT
W.
OF FOREST STANDS IN THE NORTHERN ROCKY MOUNTAINS}

Russell T. Graham and Jonalea R. Tonn

USDA Forest Service Research Paper INT-255

INTERMOUNTAIN FOREST AND RANGE EXPERIMENT STATION

Forest Service, U.S. Department of Agriculture

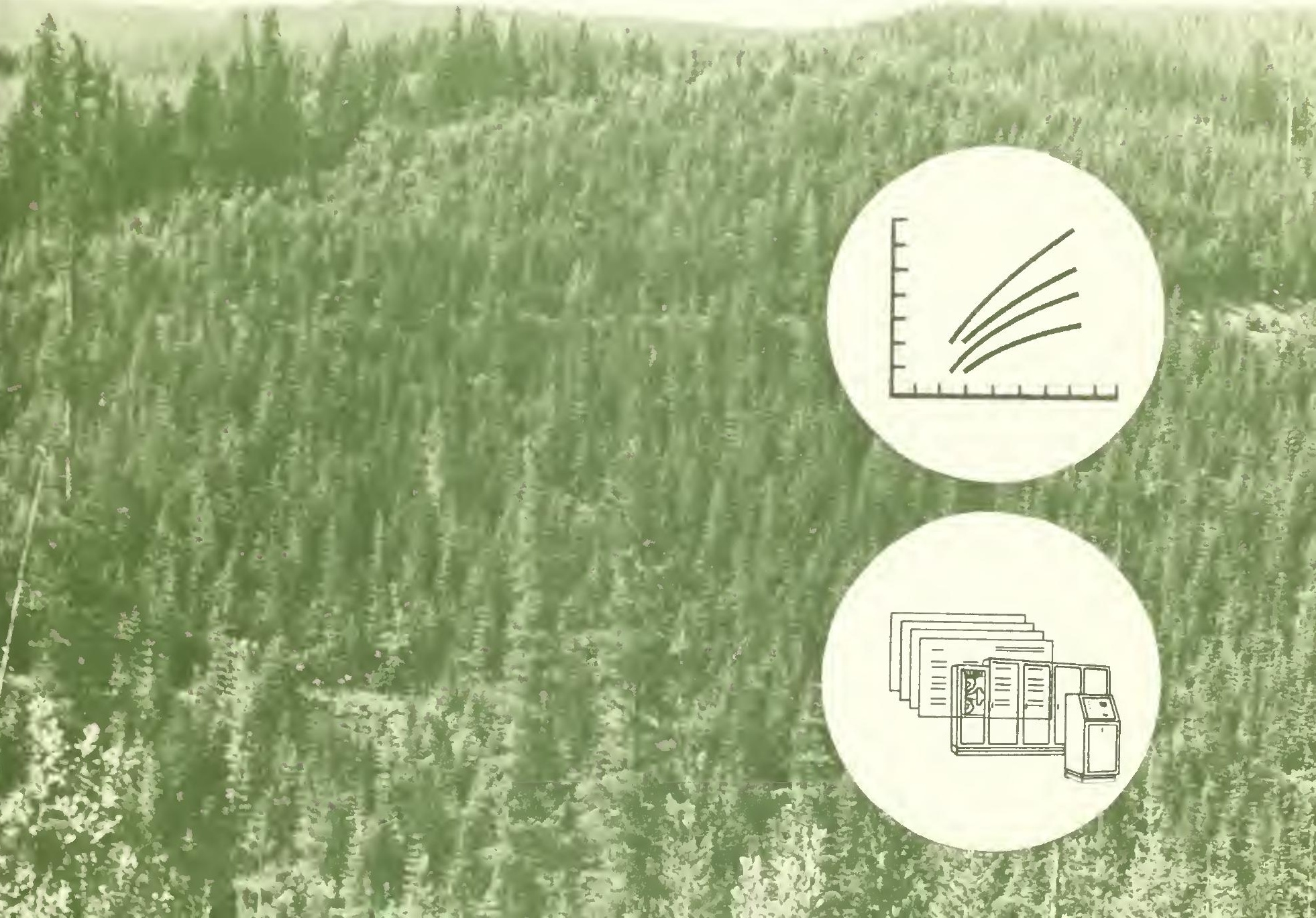




\section{THE AUTHORS}

RUSSELL T. GRAHAM is a research forester working in the silviculture of the cedar, hemlock, grand fir habitats project at the Forestry Sciences Laboratory, Moscow, Idaho. He began his career with the Eorest Service in 1973 as a forester on the Bitterroot National Forest and joined the Intermountain station in 1975. He received his B.S. in forestry from the University of Montana and his M.S. in forestry from the University of Idaho.

JONALEA R. TONN is a forester working in the silviculture of the cedar, hemlock, grand fir habitats project at the Forestry Sciences Laboratory, Moscow, Idaho. She began working for the Intermountain station in 1974. She received a B.S. and an M.F. both in forestry from the University of Idaho.

\section{ACKNOWLEDGMENT}

The authors acknowledge Region 1, Division of Timber Management, which had original responsibility for the study. staff there prepared the study plan, set up the mechanics of the system, and arranged for cooperation with the Intermountain Forest and Range Experiment station. 
CASE STUDY: GROWTH AND DEVELOPMENT OF FOREST STANDS

\section{IN THE NORTHERN ROCKY MOUNTAINS}

Russell T. Graham and Jonalea R. Tonn 


\section{RESEARCH SUMMARY}

Tree diameter and height growth were compared for nine habitat types. Ten-year aiameter growth was highly variable among habitat types as was 10-year height growth. High variation in both diameter and height growth also occurred within habitat types. Thirty-six local yield tables are presented, one for each stand in the study.

\section{CONTENTS}

INTRODUCTION. . . . . . . . . . . . . . . . . . . . 1

ANALYSIS. . . . . . . . . . . . . . . . . . 4

RESULTS. . . . . . . . . . . . . . . . . . . . 4

Diameter Growth. . . . . . . . . . . . . . . . 4

Height Growth . . . . . . . . . . . . . . . . 7

stand Yields. . . . . . . . . . . . . . . . 7

DISCUSSION. . . . . . . . . . . . . . . . . . . 7

PUBLICATIONS CITED. . . . . . . . . . . . . . . . . 9

APPENDIX ......................... . . . . . . . . . 
Information on growth and yield of managed stands plays a major role in both stand prescription preparation and timber management planning. Stand characteristics at various stages of development are used to plan intermediate cuttings as well as the timing of the final harvest. But for many Northern Rockies habitat types (Daubenmire and Daubenmire 1968) used in forest planning, there are insufficient data on forest growth and yield.

To strengthen this data base, permanent sample plots were established in 36 stands on 11 forests during 1970 and 1971 with the intention of measuring the results of management practices; therefore, thinned stands and stands scheduled for thinning were chosen. These study stands, located throughout western "lontana, northern Idaho, and eastern Washington (see fig. 1), were not selected with any randomness or experimental design in mind. In 1975 and 1976 the plots were remeasured, resulting in 5 years of growth information.

The study encompasses a wide geographical area consisting of many climatic conditions. The stand elevations range from $3,000 \mathrm{ft}(914 \mathrm{~m})$ to $7,000 \mathrm{ft}(2134 \mathrm{~m})$. The slopes and aspects of the stands are also quite variable (table 1).

Because of the diverse growing sites represented, the vegetation varies within the study areas. The tree species represented include:

Abies grandis (Doug1.) Lindl. Pseudotsuga menziesii var. qlauca (Beissn.) Franco Pinus ponderosa Laws. var. ponderosa Pinus contorta Dougl. Tsuga heterophylla (Raf.) Sarg. Thuja plicata Donn Larix occidentalis Nutt.

The habitat types represented include:

Tsuga heterophylla/Pachistima myrsinites western hemlock/pachistima myrsinites

Pinus ponderosa/Symphoricarpos albus ponderosa pine/snowberry

Pseudotsuga menziesii/Calamagrostis rubescensarctostaphylos uva-ursi Douglas-fir/pinegrass-kinnikinnick

Abies grandis/Pachistima myrsinites grand fir/pachistima myrsinites

Pseudotsuga menziesii/symphoricarpos albus Douglas-fir/snowberry

Abies lasiocarpa/Pachistima myrsinites sub-alpine fir/pachistima myrsinities

Abies lasiocarpa/Vaccinium scoparium sub-alpine fir/grouse whortleberry grand fir

Douglas-fir ponderosa pine lodgepole pine western hemlock western redcedar western larch
Tshe/Pamy

Pipo/Syal

Psme/Caru-aruv

Abgr/Pamy

Psme/Syal

Abla/Pamy

Abla/Vasc

Psme/Phma 


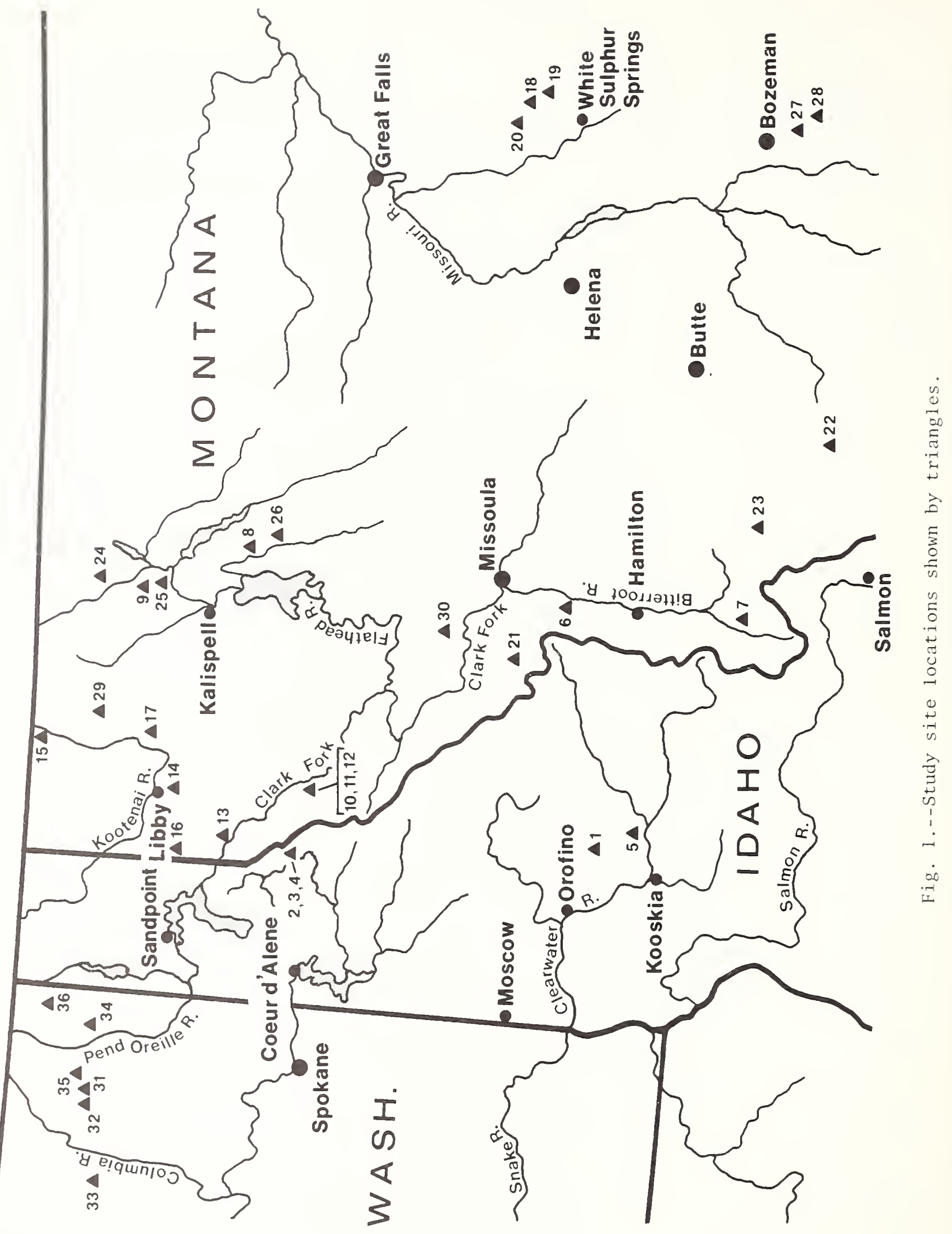




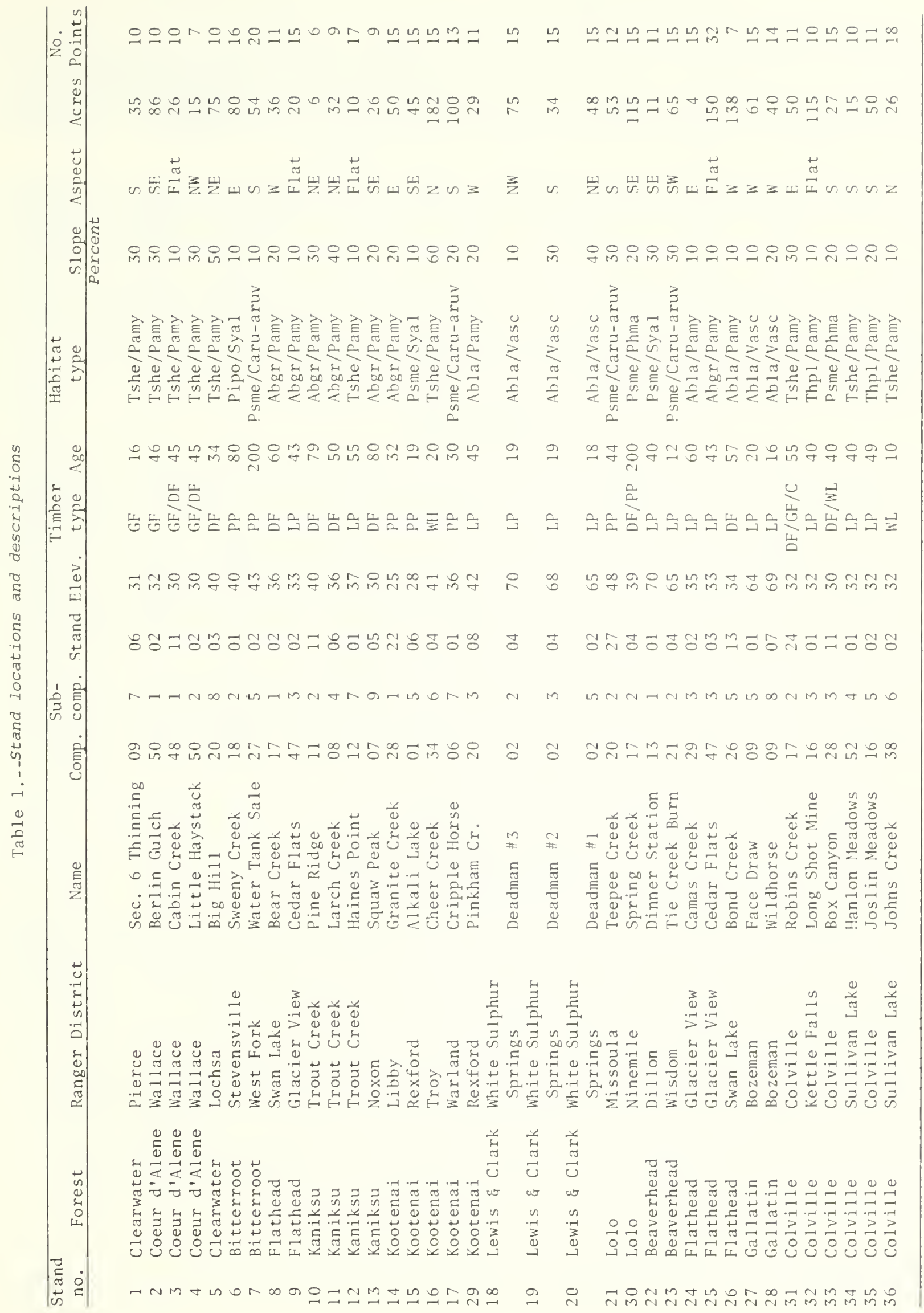


Since there were no criteria for stand selection other than past management or planned future management, many different types of stands were selected. Both stand size and number of sample points per stand were not fixed for the study. Stand age was quite variable, ranging from 10 years to 200 years (table 1 ).

As would be expected, stand characteristics differ for each stand. Vean stand diameters range from 0.9 to 16.3 inches $(2.29$ to $41.4 \mathrm{~cm})$ (table 2). Both trees per acre and accretion ${ }^{1}$ vary from stand to stand. Because of ingrowth and/or mortality, mean diameters in some of the stands are smaller for the second measurement.

Because of the nature of the data and the lack of an appropriate experimental design, we are presenting the results as a case study. The results are for the stands examined and the data should be extrapolated to other stands and conditions with caution.

\section{ANALYSIS}

The analysis involved summarizing data for the 36 stands and making appropriate comparisons. When each stand was chosen for inclusion in the study, the crop trees were tagged and permanently marked. To make valid comparisons between the first and second measurement, only crop trees were used in the thinned stands. In the unthinned stands, the entire stand as it existed was used. Diameter growth and height growth were converted to 10 -year periods for ease of comparison. Incomplete records--that is, trees dying during the study--were dropped from the diameter and height growth calculations. A least squares mean separation procedure (SAS Institute 1979) was used to separate the growth means for the habitat types.

To better show the impact of the diameter and height growth changes, each stand was projected into the future using a stand prognosis model (Stage 1973). These projections are the best estimate possible for the potential growth of the stands.

\section{RESULTS}

\section{Diameter Growth}

Tree size and age are very important in determining tree growth rates. Therefore, to make comparisons among habitat types, the diameter growth means were adjusted for both age and diameter of the trees at the time of the first measurement. In the analysis of covariance, age and initial diameter were both significant $(\mathrm{P}<.05)$ in explaining diameter growth. These adjusted growth means can be compared allowing for different tree ages and diameters.

The Pipo/syal h.t. had the smallest adjusted 10-year diameter growth mean, 0.68 inches $(1.73 \mathrm{~cm})$. The Abla/Pamy h.t. had the largest 10-year diameter growth mean, 4.26 inches $(10.82 \mathrm{~cm}$ ) (table 3 ). The adjusted means can be separated into distinct habitat type groups based on 10-year diameter growth using the least squares mean separation procedure. These were: (1) Pipo/Syal; (2) Abgr/Pamy, Thpl/Pamy; (3) Psme/Phma, Tshe/Pamy, Psme/Syal, Abla/Vasc, Psme/Caru-Aruv; and (4) the Abla/Pamy h.t.

\footnotetext{
${ }^{1}$ Periodic annual cu ft volume growth based on 5-year period excluding ingrowtn.
} 


\begin{tabular}{|c|c|c|c|c|c|c|c|c|c|c|}
\hline \multirow[b]{2}{*}{ St and } & \multicolumn{2}{|c|}{$\bar{x}$ d.b.h. } & \multicolumn{2}{|c|}{$\bar{x}$ height } & \multicolumn{2}{|c|}{ Basal area/acre } & \multicolumn{2}{|c|}{ Trees/acre } & \multicolumn{2}{|c|}{ Accretion 1} \\
\hline & First & Second & First & Second & First & Second & First & Second & First & Second \\
\hline & \multicolumn{2}{|c|}{ Inches } & \multicolumn{2}{|c|}{ Feet } & \multicolumn{2}{|c|}{$F t^{2}$} & \multirow[b]{3}{*}{300} & \multirow[b]{3}{*}{233} & \multicolumn{2}{|c|}{$F t^{3}$} \\
\hline & & & Thinn & stands & \multicolumn{2}{|c|}{ (crop trees) } & & & \multirow[b]{2}{*}{3} & \multirow[b]{2}{*}{19} \\
\hline 1. 0505706 & 0.9 & 2.4 & 6.0 & 13.1 & 1 & 8 & & & & \\
\hline 2. 1106102 & 8.1 & 9.9 & 48.5 & 56.4 & 56 & 54 & 157 & 101 & 192 & 142 \\
\hline 3. 1106111 & 6.9 & 7.7 & 46.5 & 51.4 & 78 & 84 & 304 & 260 & 135 & 172 \\
\hline 4. 1106202 & 8.6 & 9.4 & 62.5 & 64.0 & 103 & 106 & 253 & 219 & 177 & 229 \\
\hline 5. 1605803 & 8.0 & 10.3 & 51.7 & 58.5 & 34 & 36 & 97 & 62 & 124 & 181 \\
\hline 7. 2503502 & 12.3 & 8.8 & 59.5 & 37.1 & 39 & 46 & 48 & 109 & 33 & 40 \\
\hline 8. 2510102 & 8.6 & 10.1 & 51.3 & 58.3 & 73 & 73 & 183 & 131 & 82 & 121 \\
\hline 9. 2510302 & 5.8 & 6.7 & 32.6 & 40.6 & 24 & 25 & 130 & 101 & 29 & 30 \\
\hline 10. 2513211 & 10.0 & 10.4 & 81.5 & 85.3 & 147 & 147 & 267 & 249 & 117 & 104 \\
\hline 11. 2513406 & 6.8 & 7.6 & 47.6 & 52.2 & 83 & 61 & 246 & 192 & 71 & 87 \\
\hline 12. 2513701 & 4.7 & 5.6 & 40.0 & 40.3 & 46 & 48 & 381 & 286 & 143 & 145 \\
\hline 13. 2513905 & 16.3 & 13.5 & 98.5 & 65.5 & 93 & 91 & 65 & 91 & 78 & 67 \\
\hline 14. 2514122 & 8.5 & 8.5 & 42.6 & 38.7 & 41 & 45 & 105 & 113 & 135 & 80 \\
\hline 15. 2514506 & 5.1 & 7.3 & 24.5 & 31.1 & 18 & 16 & 124 & 55 & 24 & 60 \\
\hline 16. 2514604 & 2.0 & 3.2 & 15.9 & 20.9 & 6 & 11 & 260 & 200 & 11 & 18 \\
\hline 17. 2514701 & 6.1 & 7.6 & 24.8 & 32.3 & 40 & 50 & 196 & 159 & 159 & 130 \\
\hline 18. 2515204 & 2.1 & 3.2 & 11.5 & 15.6 & 9 & 22 & 380 & 380 & 30 & 55 \\
\hline 19. 2515304 & 1.8 & 2.9 & 10.8 & 14.8 & 6 & 16 & 340 & 340 & 21 & 46 \\
\hline 20. 2515502 & 1.6 & 2.8 & 8.8 & 13.1 & 4 & 12 & 300 & 300 & 13 & 31 \\
\hline 22. 3002101 & 4.1 & 4.8 & 22.0 & 24.3 & 37 & 46 & 395 & 370 & 79 & 141 \\
\hline 23. 3002204 & 0.6 & 1.9 & 5.0 & 9.5 & 1 & 5 & 280 & 280 & 3 & 17 \\
\hline 24. 3010302 & 4.7 & 5.6 & 38.3 & 39.4 & 31 & 34 & 256 & 199 & 97 & 49 \\
\hline 25. 3010303 & 6.7 & 6.5 & 43.3 & 42.0 & 32 & 65 & 129 & 286 & 37 & 138 \\
\hline 26. 3010513 & 10.5 & 12.1 & 59.9 & 60.1 & 60 & 60 & 99 & 75 & 85 & 199 \\
\hline 27. 3011501 & 3.7 & 5.2 & 17.6 & 26.1 & 32 & 47 & 433 & 315 & 72 & 55 \\
\hline 28. 3011807 & 2.3 & 3.6 & 12.4 & 17.4 & 7 & 29 & 257 & 407 & 28 & 66 \\
\hline 29. 3014308 & 5.5 & 6.3 & 37.9 & 40.0 & 39 & 45 & 237 & 208 & 58 & 166 \\
\hline 31.4607224 & 6.3 & 6.6 & 33.7 & 37.1 & 34 & 42 & 159 & 178 & 67 & 88 \\
\hline 32. 4607301 & 7.6 & 8.3 & 56.4 & 63.3 & 38 & 46 & 119 & 122 & 47 & 77 \\
\hline 33. 4607311 & 8.8 & 10.5 & 48.1 & 55.5 & 32 & 33 & 75 & 55 & 74 & 79 \\
\hline 34. 4607401 & 8.1 & 8.8 & 55.3 & 61.6 & 32 & 28 & 89 & 67 & 51 & 48 \\
\hline 35. 4607502 & 7.8 & 8.6 & 67.2 & 69.4 & 55 & 57 & 164 & 141 & 91 & 118 \\
\hline 36. 4607602 & 1.7 & 3.4 & 11.5 & 20.1 & 3 & 11 & 217 & 173 & 11 & 22 \\
\hline & & & & thinned & stands & & & & & \\
\hline 6. 2503201 & 11.3 & 9.2 & 47.2 & 39.0 & 53 & 88 & 76 & 191 & 62 & 38 \\
\hline 21.2516227 & 4.3 & 4.4 & 23.4 & 22.2 & 98 & 106 & 977 & 979 & 118 & 113 \\
\hline 30.3016204 & 6.2 & 7.8 & 34.0 & 47.5 & 108 & 98 & 508 & 295 & 93 & 95 \\
\hline
\end{tabular}

${ }^{2}$ Based on 5 years preceding measurement. 
Table 3.-- 10-year diameter growth (inches) by habitat type and stand

\begin{tabular}{|c|c|c|c|c|c|c|}
\hline \multirow[b]{2}{*}{ Habitat type } & \multirow[b]{2}{*}{ Forest } & \multirow[b]{2}{*}{ Stand } & \multicolumn{2}{|c|}{ Habitat type } & \multicolumn{2}{|c|}{ Stand } \\
\hline & & & Unadjusted & Adjusted $^{1}$ & Unadjusted & Adjusted ${ }^{1}$ \\
\hline Pipo/Syal & Bitterroot & 6 & 0.57 & $0.68 \mathrm{a}$ & 0.57 & 0.49 \\
\hline \multirow[t]{7}{*}{ Abgr/Pamy } & Flathead & 8 & 1.45 & $1.62 \mathrm{~b}$ & 2.24 & 2.34 \\
\hline & Flathead & 9 & & & 1.08 & 1.17 \\
\hline & Kaniksu & 10 & & & .73 & 1.18 \\
\hline & Kaniksu & 11 & & & 1.42 & 1.53 \\
\hline & Kaniksu & 13 & & & 1.13 & 1.20 \\
\hline & Kootenai & 14 & & & 1.92 & 1.65 \\
\hline & Flathead & 25 & & & 1.76 & 1.81 \\
\hline \multirow[t]{2}{*}{ Thp1/Pamy } & Colville & 35 & 1.51 & $1.47 \mathrm{ab}$ & 1.42 & 1.50 \\
\hline & Colville & 32 & & & 1.65 & 1.59 \\
\hline \multirow[t]{2}{*}{ Psme/Phma } & Lolo & 30 & 2.41 & $2.97 \mathrm{~cd}$ & 2.09 & 2.44 \\
\hline & Colville & 33 & & & 3.02 & 2.81 \\
\hline \multirow[t]{10}{*}{ Tshe/Pamy } & Clearwater & 1 & 2.69 & $2.25 \mathrm{c}$ & 6.14 & 6.07 \\
\hline & Coeur d'Alene & 2 & & & 2.61 & 2.58 \\
\hline & Coeur d'Alene & 3 & & & 1.66 & 1.76 \\
\hline & Coeur d'Alene & 4 & & & 1.88 & 1.82 \\
\hline & Clearwater & 5 & & & 7.23 & 7.05 \\
\hline & Kaniksu & 12 & & & 1.81 & 2.23 \\
\hline & Kootenai & 16 & & & 2.10 & 2.02 \\
\hline & Colville & 31 & & & 2.03 & 2.13 \\
\hline & Colville & 34 & & & 1.73 & 1.62 \\
\hline & Colville & 36 & & & 3.25 & 3.07 \\
\hline \multirow[t]{2}{*}{ Psme/Syal } & Kootenai & 15 & 2.82 & $2.49 \mathrm{~cd}$ & 4.00 & 3.73 \\
\hline & Beaverhead & 22 & & & 2.56 & 2.76 \\
\hline \multirow[t]{5}{*}{$\mathrm{Abla/Vasc}$} & Lewis 品 $\mathrm{Clark}$ & 18 & 3.45 & $2.34 \mathrm{c}$ & 2.21 & 2.17 \\
\hline & Lewis \& Clark & 19 & & & 2.20 & 2.18 \\
\hline & Lewis \& Clark & 20 & & & 2.56 & 2.53 \\
\hline & Gallatin & 27 & & & 5.73 & 5.62 \\
\hline & Gallatin & 28 & & & 5.33 & 5.23 \\
\hline \multirow[t]{4}{*}{ Psme/Caru-aruv } & Bitterroot & 7 & 3.71 & $2.99 \mathrm{~d}$ & 1.28 & 1.63 \\
\hline & Kootenai & 17 & & & 2.67 & 2.42 \\
\hline & Lo 10 & 21 & & & 1.27 & 1.29 \\
\hline & Beaverhead & 23 & & & 6.66 & 6.60 \\
\hline \multirow[t]{3}{*}{ Abla/Pamy } & Flathead & 24 & 3.77 & $4.26 \mathrm{e}$ & 3.44 & 3.95 \\
\hline & Flathead & 26 & & & 4.13 & 4.15 \\
\hline & Kootenai & 29 & & & 4.19 & 4.37 \\
\hline
\end{tabular}

${ }^{1}$ Adjusted for initial diameter and age. Different letters indicate significant differences. $(\mathrm{P} \leq .05)$ 
The adjusted diameter growth means for the individual stands within habitat types had a large amount of variation (table 3). The stand diameter growth means in the Tshe/Pamy h.t., ranged from 1.62 to 7.05 inches $(4.11$ to $17.91 \mathrm{~cm})$. In the Psme/Caru-Aruv h.t., adjusted diameter growth means ranged from 1.29 to 6.60 inches $(3.28$ to $16.76 \mathrm{~cm})$. The stand diameter growth means in the other habitat types showed similar variation.

\section{Height Growth}

As with diameter growth, height growth is also dependent upon tree size and age. The 10-year height growth means were adjusted using covariance analysis for initial height and age. The growth means by habitat type were then compared using SAS least squares mean separation procedure.

The Pipo/Syal h.t. had the smallest 10-year height growth mean, 4.02 ft (1.23 m). The Thpl/Pamy h.t. had the largest height growth mean, $10 \mathrm{ft}(3.05 \mathrm{~m})$ (table 4$)$. Three habitat type groups were distinguished by height growth: (1) Pipo/Syal; (2) Abla/Pamy, Psme/Syal, Psme/Phma, Abgr/Pamy, Tshe/Pamy, Abla/Vasc; (3) Psme/Caru-Aruv, Thpl/Pamy.

Within each habitat type there is a wide range of height growth means. In the Tshe/Pamy habitat type, 10-year adjusted height growth ranged from $2.34 \mathrm{ft}(0.71 \mathrm{~m})$ to a maximum of $14.18 \mathrm{ft}(4.32 \mathrm{~m})$. Likewise in the Abgr/Pamy habitat type, adjusted height growth means ranged from $1.43 \mathrm{ft}$ to $11.19 \mathrm{ft}(0.44$ to $3.41 \mathrm{~m})$.

\section{Stand Yields}

Individual tree height and diameter growth characteristics are of less consequence to forest managers than stand growth characteristics. Therefore, we projected each stand into the future using a prognosis model (Stage 1973). This procedure produced 36 local yield tables, one for each of the stands in the study (appendix tables 5-40).

In reviewing the stand projections we feel most of them are realistic. These projections may be used to estimate future volumes of stands of similar nature. Enough information is provided so the forest manager may compare his stand conditions and descriptions to those we have sumarized.

\section{DISCUSSION}

A study of this type produces a large amount of information that can be used in many ways. Silviculturists can predict future yields of stands they feel are comparable. Timber management planners and researchers can use these data to refine yield predictions.

This study encompasses a large geographic area with many different sites. Even within habitat types many different sites exist that are reflected in the mean diameter and height growths. In relation to habitat types, we have shown a wide range of growth on the same habitat type. Some areas and habitat types we expected to have poor growth proved capable of excellent diameter and height growth, especially on the central ilontana forests. 
Table 4.-- 10-year height growth (feet) by habitat type and stand

\begin{tabular}{|c|c|c|c|c|c|c|}
\hline \multirow[b]{2}{*}{ Habitat type } & \multirow[b]{2}{*}{ Forest } & \multirow[b]{2}{*}{ Stand } & \multicolumn{2}{|c|}{ Habitat type } & \multicolumn{2}{|c|}{ Stand } \\
\hline & & & Unadjusted & Adjusted ${ }^{1}$ & Unadjusted & Adjusted ${ }^{1}$ \\
\hline Pipo/Syal & Bitterroot & 6 & 3.40 & $4.02 \mathrm{a}$ & 3.4 & 3.35 \\
\hline \multirow[t]{3}{*}{ Abla/Pamy } & Flathead & 24 & 5.00 & $6.67 \mathrm{ab}$ & 3.3 & 2.64 \\
\hline & Flathead & 26 & & & 3.1 & 3.62 \\
\hline & Kootenai & 29 & & & 8.1 & 7.39 \\
\hline \multirow[t]{2}{*}{ Psme/Phma } & Lolo & 30 & 6.10 & $7.23 \mathrm{abc}$ & 2.6 & 3.35 \\
\hline & Colville & 33 & & & 12.5 & 12.45 \\
\hline \multirow[t]{2}{*}{ Psme/Syal } & Kootenai & 15 & 6.60 & $6.77 \mathrm{ab}$ & 13.7 & 12.21 \\
\hline & Beaverhead & 22 & & & 5.0 & 3.40 \\
\hline \multirow[t]{5}{*}{$\mathrm{Abla} / \mathrm{Vasc}$} & Lewis \& Clark & 18 & 7.00 & $8.65 \mathrm{bc}$ & 8.3 & 6.19 \\
\hline & Lewis \& Clark & 19 & & & 8.1 & 5.95 \\
\hline & Lewis \& Clark & 20 & & & 8.6 & 6.26 \\
\hline & Gallatin & 27 & & & 16.5 & 14.69 \\
\hline & Gallatin & 28 & & & 11.2 & 9.09 \\
\hline \multirow[t]{7}{*}{ Abgr/Pamy } & Flathead & 8 & 7.10 & $7.48 \mathrm{abc}$ & 10.7 & 10.90 \\
\hline & Flathead & 9 & & & 6.1 & 5.36 \\
\hline & Kaniksu & 10 & & & 7.4 & 9.10 \\
\hline & Kaniksu & 11 & & & 7.8 & 7.71 \\
\hline & Kaniksu & 13 & & & 5.7 & 8.43 \\
\hline & Kootenai & 14 & & & 11.9 & 11.19 \\
\hline & Flathead & 25 & & & 1.8 & 1.43 \\
\hline \multirow[t]{10}{*}{ Tshe/Pamy } & Clearwater & 1 & 8.80 & $8.03 \mathrm{bc}$ & 10.6 & 8.24 \\
\hline & Coeur d'Alene & 2 & & & 10.8 & 10.86 \\
\hline & Coeur d'Alene & 3 & & & 10.8 & 10.53 \\
\hline & Coeur d'Alene & 4 & & & 7.6 & 8.30 \\
\hline & Clearwater & 5 & & & 9.0 & 9.05 \\
\hline & Kaniksu & 12 & & & 3.0 & 2.34 \\
\hline & Kootenai & 16 & & & 7.8 & 5.98 \\
\hline & Colville & 31 & & & 14.0 & 12.99 \\
\hline & Colville & 34 & & & 13.9 & 14.18 \\
\hline & Colville & 36 & & & 13.6 & 11.51 \\
\hline \multirow[t]{4}{*}{ Psme/Caru-aurv } & Bitterroot & 7 & 10.00 & $8.97 \mathrm{c}$ & 6.2 & 6.72 \\
\hline & Kootenai & 17 & & & 13.0 & 11.59 \\
\hline & Lolo & 21 & & & 8.8 & 7.73 \\
\hline & Beaverhead & 23 & & & 10.0 & 7.59 \\
\hline \multirow[t]{2}{*}{ Thpl/Pamy } & Colville & 32 & 11.10 & $10.00 \mathrm{c}$ & 15.5 & 1.58 \\
\hline & Colville & 35 & & & 8.2 & 9.18 \\
\hline
\end{tabular}

${ }^{I}$ Adjusted for initial height and age. Different letters indicate significant differences. $(\mathrm{P}<.05)$ 
Many variables influence tree growth, making it difficult to compare the growth of the stands and the habitat types. We have a limited sample of the numerous sites available in the study area. Using a covariance analysis, with both tree size and age as covariates, we allowed for many differences that exist among the stands. These comparisons and yield tables provide a reference point for future management activities on these habitat types.

\section{PUBLICATIONS CITED}

Daubenmire, R., and Jean B. Daubenmire.

1968. Forest vegetation of eastern Washington and northern Idaho. Wash. Agric. Exp. Stn. Tech. Bull. 60, 104 p. Pullman, Wash.

SAS Institute.

1979. SAS User's Guide 1979 edition. SAS Institute Inc. Post Office Box 10066 , Raleigh, North Carolina 27605.

Stage, A. R.

1973. Prognosis model for stand development. USDA For. Serv. Res. Pap. INT-127, 32 p. Intermt. For. and Range Exp. Stn., Ogden, Utah. 


\section{APPENDIX}

Note: For all yield tables in the appendix, merchantable volume was measured to a 4 -inch top. 
Table 5.--Yield table for Stand 1, Clearwater National Forest, Pierce Ranger District, Section 6 Thinning

\begin{tabular}{|c|c|c|c|c|c|c|c|c|c|c|}
\hline \multirow[b]{2}{*}{ Age } & \multirow[b]{2}{*}{ Trees/acre } & \multirow[b]{2}{*}{$\mathrm{BA} / \mathrm{acre}$} & \multirow[b]{2}{*}{$\mathrm{CCF}$} & \multicolumn{3}{|c|}{ Volume per acre } & \multirow[b]{2}{*}{ Accretion } & \multirow[b]{2}{*}{ Yortality } & \multirow[b]{2}{*}{$\bar{x}$ d.b.h. } & \multirow[b]{2}{*}{$\bar{x}$ height } \\
\hline & & & & $\begin{array}{l}\text { Total } \\
\mathrm{ft}^{3} \\
\end{array}$ & $\begin{array}{c}\text { Merchantable } \\
\mathrm{ft}^{3}\end{array}$ & $\begin{array}{c}\text { Scribner } \\
\text { bd.ft. }\end{array}$ & & & & \\
\hline & & $F t^{2}$ & & & & & $F t^{3}$ & $F t^{3}$ & Inches & Feet \\
\hline 21 & 233 & 8 & 30 & 59 & 0 & 0 & 19 & 1 & 2.4 & 13.1 \\
\hline 31 & 184 & 18 & 49 & 236 & 50 & 0 & 33 & 3 & 4.2 & 24.5 \\
\hline 41 & 158 & 30 & 65 & 535 & 432 & 170 & 47 & 6 & 5.9 & 34.9 \\
\hline 51 & 141 & 43 & 79 & 946 & 854 & 1,726 & 69 & 7 & 7.5 & 45.4 \\
\hline 61 & 129 & 61 & 96 & 1,564 & 1,444 & 5,086 & 83 & 9 & 9.3 & 56.0 \\
\hline 71 & 121 & 79 & 112 & 2,309 & 2,174 & 8,756 & 106 & 12 & 10.9 & 66.0 \\
\hline 81 & 115 & 99 & 128 & 3,242 & 3,079 & 13,487 & 137 & 17 & 12.6 & 75.7 \\
\hline 91 & 109 & 123 & 145 & 4,437 & 4,192 & 19,240 & 137 & 24 & 14.4 & 84.8 \\
\hline 101 & 103 & 143 & 159 & 5,571 & 5,236 & 24,361 & 168 & 30 & 16.0 & 93.3 \\
\hline 111 & 98 & 168 & 176 & 6,951 & 6,485 & 30,609 & 176 & 38 & 17.7 & 101.2 \\
\hline 121 & 93 & 191 & 190 & 8,335 & 7,695 & 36,367 & 0 & 0 & 19.4 & 108.6 \\
\hline
\end{tabular}

Table 6.--Yield table for Stand 2, Coeur d'Alene National Forest, wallace Ranger District, Berlin Gulch

\begin{tabular}{|c|c|c|c|c|c|c|c|c|c|c|}
\hline \multirow[b]{2}{*}{ Age } & \multirow[b]{2}{*}{ Trees/acre } & \multirow[b]{2}{*}{$\mathrm{BA} / \mathrm{acre}$} & \multirow[b]{2}{*}{ CCF } & \multicolumn{3}{|c|}{ Volume per acre } & \multirow[b]{2}{*}{ Accretion } & \multirow[b]{2}{*}{ Mortality } & \multirow[b]{2}{*}{$\bar{x}$ d.b.h. } & \multirow[b]{2}{*}{$\bar{x}$ height } \\
\hline & & & & $\begin{array}{c}\text { Total } \\
\mathrm{ft}^{3}\end{array}$ & $\begin{array}{c}\text { Merchantable } \\
\mathrm{ft}^{3}\end{array}$ & $\begin{array}{c}\text { Scribner } \\
\text { bd.ft. }\end{array}$ & & & & \\
\hline & & $F t^{2}$ & & & & & $F t^{3}$ & $F t^{3}$ & Inches & Feet \\
\hline 51 & 101 & 54 & 81 & 1,538 & 1,293 & 4,427 & 142 & 9 & 9.9 & 56.4 \\
\hline 61 & 94 & 88 & 111 & 2,859 & 2,431 & 10,163 & 177 & 17 & 13.0 & 69.9 \\
\hline 71 & 89 & 122 & 138 & 4,460 & 3,782 & 17,265 & 203 & 27 & 15.9 & 82.2 \\
\hline 81 & 84 & 155 & 160 & 6,219 & 5,234 & 24,864 & 230 & 39 & 18.4 & 93.7 \\
\hline 91 & 79 & 188 & 181 & 8,129 & 6,781 & 32,995 & 243 & 52 & 20.9 & 104.3 \\
\hline 101 & 74 & 219 & 201 & 10,039 & 8,305 & 40,997 & 260 & 65 & 23.3 & 114.1 \\
\hline 111 & 69 & 250 & 225 & 11,981 & 9,868 & 49,217 & 263 & 80 & 25.8 & 123.1 \\
\hline 121 & 65 & 280 & 247 & 13,817 & 11,363 & 57,008 & 247 & 94 & 28.2 & 131.3 \\
\hline 131 & 61 & 304 & 264 & 15,350 & 12,656 & 63,693 & 232 & 106 & 30.3 & 138.8 \\
\hline 141 & 56 & 324 & 279 & 16,607 & 13,785 & 69,402 & 221 & 117 & 32.4 & 145.7 \\
\hline 151 & 53 & 341 & 290 & 17,642 & 14,773 & 74,281 & 0 & 0 & 34.4 & 152.0 \\
\hline
\end{tabular}

Table 7.--Yield table for Stand 3, Coeur d'Alene National Forest, Wallace Ranger District Cabin Creek

\begin{tabular}{|c|c|c|c|c|c|c|c|c|c|c|}
\hline \multirow[b]{2}{*}{ Age } & \multirow[b]{2}{*}{ Trees/acre } & \multirow[b]{2}{*}{$\mathrm{BA} / \mathrm{acre}$} & \multirow[b]{2}{*}{ CCF } & \multicolumn{3}{|c|}{ Volume per acre } & \multirow[b]{2}{*}{ Accretion } & \multirow[b]{2}{*}{ Mortality } & \multirow[b]{2}{*}{$\bar{x}$ d.b.h. } & \multirow[b]{2}{*}{$\bar{x}$ height } \\
\hline & & & & $\begin{array}{c}\text { Total } \\
\mathrm{ft}^{3} \\
\end{array}$ & $\begin{array}{c}\text { Merchantable } \\
\mathrm{ft}^{3}\end{array}$ & $\begin{array}{c}\text { Scribner } \\
\text { bd.ft. }\end{array}$ & & & & \\
\hline & & $F t^{2}$ & & & & & $F t^{3}$ & $F t^{3}$ & Inches & Feet \\
\hline 50 & 260 & 84 & 130 & 2,020 & 1,670 & 3,567 & 172 & 15 & 7.7 & 51.4 \\
\hline 60 & 239 & 125 & 162 & 3,591 & 3,094 & 10,830 & 210 & 21 & 9.8 & 63.5 \\
\hline 70 & 224 & 167 & 189 & 5,484 & 4,787 & 19,565 & 229 & 31 & 11.7 & 74.6 \\
\hline 80 & 211 & 203 & 214 & 7,465 & 6,530 & 29,149 & 261 & 43 & 13.3 & 84.7 \\
\hline 90 & 200 & 241 & 243 & 9,650 & 8,450 & 39,645 & 288 & 56 & 14.9 & 94.0 \\
\hline 100 & 188 & 277 & 270 & 11,965 & 10,414 & 50,280 & 286 & 71 & 16.4 & 102.5 \\
\hline 110 & 178 & 308 & 292 & 14,112 & 12,209 & 59,954 & 288 & 86 & 17.8 & 110.3 \\
\hline 120 & 167 & 334 & 311 & 16,138 & 13,873 & 69,344 & 286 & 100 & 19.1 & 117.5 \\
\hline 130 & 158 & 357 & 327 & 18,002 & 15,401 & 77,899 & 282 & 113 & 20.4 & 124.1 \\
\hline 140 & 148 & 377 & 339 & 19,694 & 16,781 & 85,590 & 272 & 125 & 21.6 & 130.2 \\
\hline 150 & 139 & 393 & 349 & 21,163 & 17,974 & 92,360 & 0 & 0 & 22.7 & 135.9 \\
\hline
\end{tabular}


Table 8.--Yield table for Stand 4, Coeur d'Alene National Forest, Wallace Ranger District, Little Haystack

Volume per acre

Total Merchantable Scribner

Age Trees/acre BA/acre CCF $\mathrm{ft}^{3} \quad \mathrm{ft}^{3} \quad$ bd.ft. Accretion Mortality $\bar{x} \mathrm{~d} . \mathrm{b} . \mathrm{h}$. $\bar{x}$ height

\begin{tabular}{|c|c|c|c|c|c|c|c|c|c|c|}
\hline & & $F t^{2}$ & & & & & $F t^{3}$ & $F t^{3}$ & Inches & Feet \\
\hline 50 & 219 & 106 & 149 & 3,041 & 2,689 & 7,566 & 229 & 19 & 9.4 & 64.0 \\
\hline 60 & 205 & 156 & 183 & 5,143 & 4,570 & 17,780 & 259 & 29 & 11.8 & 75.6 \\
\hline 70 & 194 & 203 & 213 & 7,444 & 6,595 & 28,617 & 266 & 43 & 13.8 & 85.7 \\
\hline 80 & 183 & 241 & 241 & 9,672 & 8,511 & 38,798 & 289 & 58 & 15.5 & 94.7 \\
\hline 90 & 172 & 278 & 267 & 11,979 & 10,454 & 48,948 & 311 & 76 & 17.2 & 102.9 \\
\hline 100 & 162 & 314 & 292 & 14,333 & 12,350 & 58,972 & 308 & 97 & 18.9 & 110.4 \\
\hline 110 & 151 & 343 & 311 & 16,444 & 13,985 & 67,465 & 300 & 117 & 20.4 & 117.3 \\
\hline 120 & 140 & 366 & 326 & 18,275 & 15,371 & 74,631 & 289 & 135 & 21.9 & 123.8 \\
\hline 130 & 130 & 384 & 335 & 19,816 & 16,493 & 80,179 & 291 & 148 & 23.3 & 129.9 \\
\hline 140 & 120 & 400 & 345 & 21,251 & 17,533 & 85,170 & 266 & 161 & 24.7 & 135.6 \\
\hline 150 & 110 & 410 & 349 & 22,301 & 18,269 & 88,368 & 0 & 0 & 26.1 & 141.1 \\
\hline
\end{tabular}

Table 9.--Yield table for Stand 5, Clearwater National Forest, Lochsa Ranger District, Big Hill

\begin{tabular}{|c|c|c|c|c|c|c|c|c|c|c|}
\hline \multirow[b]{2}{*}{ Age } & \multirow[b]{2}{*}{ Trees/acre } & \multirow[b]{2}{*}{$\mathrm{BA} / \mathrm{acre}$} & \multirow[b]{2}{*}{ CCF } & \multicolumn{3}{|c|}{ Volume per acre } & \multirow[b]{2}{*}{ Accretion } & \multirow[b]{2}{*}{ Mortality } & \multirow[b]{2}{*}{$\bar{x}$ d.b.h. } & \multirow[b]{2}{*}{$\bar{x}$ height } \\
\hline & & & & $\begin{array}{l}\text { Total } \\
\mathrm{ft}^{3}\end{array}$ & $\begin{array}{l}\text { Merchantable } \\
\mathrm{ft}^{3}\end{array}$ & $\begin{array}{l}\text { Scribner } \\
\text { bd.ft: }\end{array}$ & & & & \\
\hline & & $F t^{2}$ & & & & & $F t^{3}$ & $F t^{3}$ & Inches & Feet \\
\hline 39 & 62 & 36 & 51 & 863 & 851 & 3,015 & 181 & 5 & 10.3 & 58.5 \\
\hline 49 & 59 & 96 & 97 & 2,623 & 2,512 & 10,191 & 244 & 13 & 17.2 & 75.6 \\
\hline 59 & 56 & 160 & 138 & 4,930 & 4,432 & 13,980 & 271 & 26 & 22.9 & 88.0 \\
\hline 69 & 53 & 219 & 166 & 7,372 & 6,168 & 14,077 & 316 & 42 & 27.5 & 98.1 \\
\hline 79 & 50 & 280 & 192 & 10,110 & 7,859 & 12,231 & 351 & 62 & 32.0 & 106.8 \\
\hline 89 & 47 & 339 & 220 & 13,008 & 9,433 & 10,116 & 341 & 85 & 36.3 & 114.3 \\
\hline 99 & 44 & 386 & 244 & 15,572 & 10,540 & 8,622 & 362 & 108 & 39.9 & 120.8 \\
\hline 109 & 42 & 430 & 265 & 18,116 & 11,407 & 9,432 & 359 & 135 & 43.6 & 126.7 \\
\hline 119 & 39 & 466 & 282 & 20,356 & 11,941 & 14,450 & 349 & 161 & 46.9 & 132.0 \\
\hline 129 & 36 & 492 & 293 & 22,231 & 12,158 & 24,537 & 319 & 188 & 50.0 & 136.8 \\
\hline 139 & 33 & 506 & 298 & 23,547 & 12,042 & 39,216 & 0 & 0 & 52.6 & 141.2 \\
\hline
\end{tabular}

Table 10.--Yield table for Stand 6, Bitterroot National Forest, Stevensville Ranger. District, Sweeny Creek

\begin{tabular}{|c|c|c|c|c|c|c|c|c|c|c|}
\hline \multirow[b]{2}{*}{ Age } & \multirow[b]{2}{*}{ Trees/acre } & \multirow[b]{2}{*}{$\mathrm{BA} / \mathrm{acre}$} & \multirow[b]{2}{*}{$\mathrm{CCF}$} & \multicolumn{3}{|c|}{ Volume per acre } & \multirow[b]{2}{*}{ Accretion } & \multirow[b]{2}{*}{ Mortality } & \multirow[b]{2}{*}{$\bar{x}$ d.b.h. } & \multirow[b]{2}{*}{$\bar{x}$ height } \\
\hline & & & & $\begin{array}{l}\text { Total } \\
\mathrm{ft}^{3}\end{array}$ & $\begin{array}{c}\text { Merchantable } \\
\mathrm{ft}^{3}\end{array}$ & $\begin{array}{c}\text { Scribner } \\
\text { bd.ft. }\end{array}$ & & & & \\
\hline & & $F t^{2}$ & & & & & $F t^{3}$ & $F t^{3}$ & Inches & Feet \\
\hline 85 & 191 & 88 & 95 & 1,964 & 1,486 & 4,790 & 38 & 11 & 9.2 & 39.0 \\
\hline 95 & 177 & 95 & 98 & 2,234 & 1,709 & 5,689 & 40 & 14 & 9.9 & 45.6 \\
\hline 105 & 165 & 98 & 98 & 2,493 & 1,930 & 6,771 & 41 & 16 & 10.4 & 50.9 \\
\hline 115 & 154 & 100 & 99 & 2,743 & 2,142 & 7,815 & 43 & 18 & 10.9 & 55.9 \\
\hline 125 & 143 & 102 & 99 & 3,000 & 2,361 & 9,029 & 42 & 20 & 11.4 & 60.5 \\
\hline 135 & 133 & 103 & 98 & 3,217 & 2,547 & 10,220 & 42 & 21 & 11.9 & 64.7 \\
\hline 145 & 124 & 105 & 98 & 3,419 & 2,721 & 11,377 & 39 & 23 & 12.5 & 68.6 \\
\hline 155 & 115 & 105 & 97 & 3,579 & 2,860 & 12,362 & 38 & 24 & 12.9 & 72.2 \\
\hline 165 & 107 & 105 & 96 & 3,725 & 2,987 & 13,273 & 37 & 24 & 13.4 & 75.4 \\
\hline 175 & 100 & 104 & 95 & 3,849 & 3,094 & 14,130 & 33 & 25 & 13.8 & 78.4 \\
\hline 185 & 93 & 103 & 93 & 3,932 & 3,168 & 14,779 & 0 & 0 & 14.2 & 81.2 \\
\hline
\end{tabular}


Table 11.--Yield table for Stand 7, Bitterroot National Forest, West Fork Ranger District, Water Tank Sale

\begin{tabular}{|c|c|c|c|c|c|c|c|c|c|c|}
\hline \multirow[b]{2}{*}{ Age } & \multirow[b]{2}{*}{ Trees/acre } & \multirow[b]{2}{*}{$\mathrm{BA} / \mathrm{acre}$} & \multirow[b]{2}{*}{$\mathrm{CCF}$} & \multicolumn{3}{|c|}{ Volume per acre } & \multirow[b]{2}{*}{ Accretion } & \multirow[b]{2}{*}{ Mortality } & \multirow[b]{2}{*}{$\bar{x}$ d.b.h. } & \multirow[b]{2}{*}{$\bar{x}$ height } \\
\hline & & & & $\begin{array}{c}\text { Total } \\
\mathrm{ft}^{3}\end{array}$ & $\begin{array}{c}\text { Merchantable } \\
\mathrm{ft}^{3}\end{array}$ & $\begin{array}{l}\text { Scribner } \\
\text { bd.ft. }\end{array}$ & & & & \\
\hline & & $F t^{2}$ & & & & & $F t^{3}$ & $F t^{3}$ & Inches & Feet \\
\hline 205 & 109 & 46 & 52 & 1,437 & 1,147 & 4,385 & 40 & 7 & 8.8 & 37.1 \\
\hline 215 & 100 & 53 & 59 & 1,765 & 1,424 & 6,018 & 51 & 8 & 9.9 & 46.4 \\
\hline 225 & 94 & 65 & 68 & 2,198 & 1,764 & 8,026 & 50 & 9 & 11.3 & 57.3 \\
\hline 235 & 89 & 72 & 73 & 2,606 & 2,109 & 10,010 & 54 & 11 & 12.2 & 64.2 \\
\hline 245 & 85 & 79 & 77 & 3,040 & 2,476 & 12,056 & 59 & 13 & 13.1 & 70.8 \\
\hline 255 & 81 & 87 & 82 & 3,503 & 2,869 & 14,246 & 58 & 15 & 14.1 & 76.6 \\
\hline 265 & 77 & 93 & 85 & 3,939 & 3,238 & 16,367 & 59 & 16 & 14.9 & 81.9 \\
\hline 275 & 74 & 99 & 89 & 4,368 & 3,605 & 18,741 & 63 & 18 & 15.7 & 86.6 \\
\hline 285 & 71 & 106 & 92 & 4,820 & 3,994 & 21,261 & 61 & 20 & 16.6 & 90.9 \\
\hline 295 & 68 & 111 & 95 & 5,232 & 4,351 & 23,615 & 63 & 22 & 17.3 & 94.9 \\
\hline 305 & 65 & 116 & 97 & 5,642 & 4,700 & 25,804 & 0 & 0 & 18.1 & 98.5 \\
\hline
\end{tabular}

Table 12.--Yield table for Stand 8, Flathead National Forest, Swan Lake Ranger District, Bear Creek

\begin{tabular}{|c|c|c|c|c|c|c|c|c|c|c|}
\hline \multirow[b]{2}{*}{ Age } & \multirow[b]{2}{*}{ Trees/acre } & \multirow[b]{2}{*}{$\mathrm{BA} / \mathrm{acre}$} & \multirow[b]{2}{*}{$\mathrm{CCF}$} & \multicolumn{3}{|c|}{ Volume per acre } & \multirow[b]{2}{*}{ Accretion } & \multirow[b]{2}{*}{ Mortality } & \multirow[b]{2}{*}{$\bar{x}$ d.b.h. } & \multirow[b]{2}{*}{$\bar{x}$ height } \\
\hline & & & & $\begin{array}{c}\text { Total } \\
\mathrm{ft}^{3}\end{array}$ & $\begin{array}{c}\text { Merchantable } \\
\mathrm{ft}^{3}\end{array}$ & $\begin{array}{l}\text { Scribner } \\
\text { bd.ft. }\end{array}$ & & & & \\
\hline & & $F t^{2}$ & & & & & $F t^{3}$ & $F t^{3}$ & Inches & Feet \\
\hline 65 & 131 & 73 & 96 & 1,772 & 1,628 & 5,224 & 121 & 8 & 10.1 & 58.3 \\
\hline 75 & 124 & 106 & 118 & 2,896 & 2,696 & 10,086 & 130 & 13 & 12.5 & 69.3 \\
\hline 85 & 118 & 136 & 134 & 4,064 & 3,712 & 15,210 & 134 & 18 & 14.5 & 78.1 \\
\hline 95 & 113 & 163 & 148 & 5,224 & 4,644 & 19,640 & 147 & 23 & 16.2 & 85.5 \\
\hline 105 & 109 & 189 & 160 & 6,465 & 5,567 & 23,779 & 163 & 29 & 17.9 & 92.0 \\
\hline 115 & 104 & 218 & 172 & 7,809 & 6,490 & 27,686 & 162 & 36 & 19.5 & 97.8 \\
\hline 125 & 100 & 242 & 182 & 9,074 & 7,296 & 30,906 & 166 & 43 & 21.0 & 102.9 \\
\hline 135 & 96 & 265 & 193 & 10,310 & 8,010 & 33,408 & 165 & 50 & 22.4 & 107.5 \\
\hline 145 & 93 & 284 & 203 & 11,460 & 8,635 & 35,476 & 175 & 57 & 23.7 & 111.8 \\
\hline 155 & 89 & 304 & 213 & 12,646 & 9,218 & 37,187 & 167 & 64 & 25.1 & 115.6 \\
\hline 165 & 85 & 320 & 220 & 13,676 & 9,648 & 38,136 & 0 & 0 & 26.2 & 119.1 \\
\hline
\end{tabular}

Table 13.--Yield table for Stand 9, Flathead National Forest, Glacier View Ranger District, Cedar Flats

\begin{tabular}{|c|c|c|c|c|c|c|c|c|c|c|}
\hline \multirow[b]{2}{*}{ Age } & \multirow[b]{2}{*}{ Trees/acre } & \multirow[b]{2}{*}{ BA/acre } & \multirow[b]{2}{*}{$\mathrm{CCF}$} & \multicolumn{3}{|c|}{ Volume per acre } & \multirow[b]{2}{*}{ Accretion } & \multirow[b]{2}{*}{ :lortality } & \multirow[b]{2}{*}{$\bar{x}$ d.b.h. } & \multirow[b]{2}{*}{$\bar{x}$ height } \\
\hline & & & & $\begin{array}{c}\text { Total } \\
\mathrm{ft}^{3}\end{array}$ & $\begin{array}{c}\text { Merchantable } \\
\mathrm{ft}^{3}\end{array}$ & $\begin{array}{l}\text { Scribner } \\
\text { bd.ft. }\end{array}$ & & & & \\
\hline & & $F t^{2}$ & & & & & $F t^{3}$ & $F t^{3}$ & Inches & Feet \\
\hline 48 & 101 & 25 & 39 & 532 & 381 & 306 & 30 & 5 & 6.7 & 40.6 \\
\hline 58 & 91 & 32 & 46 & 781 & 609 & 1,358 & 35 & 6 & 8.0 & 47.9 \\
\hline 68 & 84 & 38 & 52 & 1,075 & 837 & 2,681 & 39 & 7 & 9.1 & 54.9 \\
\hline 78 & 78 & 45 & 58 & 1,393 & 1,058 & 4,035 & 45 & 8 & 10.2 & 61.5 \\
\hline 88 & 74 & 52 & 64 & 1,756 & 1,293 & 5,110 & 52 & 11 & 11.3 & 67.8 \\
\hline 98 & 69 & 59 & 69 & 2,160 & 1,542 & 6,193 & 52 & 16 & 12.5 & 73.7 \\
\hline 108 & 65 & 64 & 73 & 2,519 & 1,752 & 6,993 & 54 & 23 & 13.5 & 79.4 \\
\hline 118 & 59 & 68 & 76 & 2,836 & 1,922 & 7,561 & 54 & 32 & 14.5 & 84.7 \\
\hline 128 & 53 & 69 & 76 & 3,058 & 2,021 & 7,777 & 53 & 43 & 15.4 & 89.7 \\
\hline 138 & 47 & 68 & 74 & 3,159 & 2,034 & 7,615 & 48 & 56 & 16.3 & 94.4 \\
\hline 148 & 40 & 64 & 70 & 3,082 & 1,932 & 6,998 & 0 & 0 & 17.2 & 98.8 \\
\hline
\end{tabular}


Table 14.--Yield table for Stand 10, Kaniksu National Forest, Trout Creek Ranger District, Pine Ridge

Volume per acre

Total Merchantable Scribner

Age Trees/acre BA/acre CCF $\mathrm{ft}^{3} \quad \mathrm{ft}^{3} \quad$ bd.ft. Accretion Mortality $\bar{x} \mathrm{~d} . \mathrm{b} . \mathrm{h}$. $\overline{\mathrm{x}}$ height

\begin{tabular}{|c|c|c|c|c|c|c|c|c|c|c|}
\hline & & $F t^{2}$ & & & & & $F t^{3}$ & $F t^{3}$ & Inches & Feet \\
\hline 84 & 249 & 147 & 171 & 5,039 & 4,435 & 18,338 & 104 & 27 & 10.4 & 85.3 \\
\hline 94 & 236 & 159 & 173 & 5,815 & 5,134 & 22,708 & 108 & 30 & 11.1 & 91.3 \\
\hline 104 & 224 & 171 & 178 & 6,595 & 5,845 & 27,122 & 100 & 34 & 11.9 & 96.8 \\
\hline 114 & 212 & 180 & 181 & 7,259 & 6,454 & 30,937 & 110 & 38 & 12.5 & 101.9 \\
\hline 124 & 202 & 190 & 185 & 7,977 & 7,140 & 35,125 & 107 & 44 & 13.1 & 106.7 \\
\hline 134 & 192 & 198 & 188 & 8,609 & 7,763 & 38,946 & 103 & 50 & 13.8 & 111.1 \\
\hline 144 & 182 & 204 & 189 & 9,138 & 8,288 & 41,975 & 108 & 56 & 14.4 & 115.2 \\
\hline 154 & 172 & 210 & 191 & 9,658 & 8,807 & 45,006 & 107 & 63 & 15.0 & 119.0 \\
\hline 164 & 162 & 215 & 191 & 10,097 & 9,258 & 47,505 & 94 & 67 & 15.6 & 122.6 \\
\hline 174 & 153 & 217 & 189 & 10,373 & 9,554 & 49,434 & 99 & 68 & 16.1 & 126.0 \\
\hline 184 & 145 & 220 & 188 & 10,678 & 9,897 & 51,568 & 0 & 0 & 16.7 & 129.3 \\
\hline
\end{tabular}

Table 15.--Yield table for Stand 11, Kaniksu National Forest, Trout Creek Ranger District, Larch Creek

\begin{tabular}{|c|c|c|c|c|c|c|c|c|c|c|}
\hline \multirow[b]{2}{*}{ Age } & \multirow[b]{2}{*}{ Trees/acre } & \multirow[b]{2}{*}{$\mathrm{BA} / \mathrm{acre}$} & \multirow[b]{2}{*}{$\mathrm{CCF}$} & \multicolumn{3}{|c|}{ Volume per acre } & \multirow[b]{2}{*}{ Accretion } & \multirow[b]{2}{*}{ Mortality } & \multirow[b]{2}{*}{$\bar{x}$ d.b.h. } & \multirow[b]{2}{*}{ height } \\
\hline & & & & $\begin{array}{l}\text { Total } \\
\mathrm{ft}^{3}\end{array}$ & $\begin{array}{c}\text { Merchantable } \\
\mathrm{ft}^{3}\end{array}$ & $\begin{array}{c}\text { Scribner } \\
\text { bd.ft. }\end{array}$ & & & & \\
\hline & & $F t^{2}$ & & & & & $F t^{3}$ & $F t^{3}$ & Inches & Feet \\
\hline 55 & 192 & 61 & 110 & 1,355 & 1,083 & 1,995 & 87 & 10 & 7.6 & 52.2 \\
\hline 65 & 177 & 84 & 124 & 2,124 & 1,835 & 5,593 & 98 & 12 & 9.3 & 63.1 \\
\hline 75 & 166 & 106 & 137 & 2,987 & 2,649 & 10,163 & 101 & 14 & 10.8 & 72.6 \\
\hline 85 & 158 & 126 & 147 & 3,855 & 3,463 & 15,074 & 111 & 18 & 12.1 & 80.9 \\
\hline 95 & 150 & 146 & 156 & 4,789 & 4,343 & 20,311 & 120 & 22 & 13.3 & 88.3 \\
\hline 105 & 143 & 166 & 165 & 5,769 & 5,276 & 25,816 & 126 & 27 & 14.6 & 94.9 \\
\hline 115 & 136 & 185 & 173 & 6,755 & 6,224 & 31,491 & 132 & 32 & 15.8 & 100.8 \\
\hline 125 & 130 & 203 & 181 & 7,753 & 7,187 & 37,341 & 128 & 37 & 16.9 & 106.2 \\
\hline 135 & 124 & 219 & 188 & 8,658 & 8,049 & 42,652 & 131 & 42 & 18.0 & 111.1 \\
\hline 145 & 118 & 233 & 195 & 9,543 & 8,889 & 47,979 & 130 & 47 & 19.0 & 115.6 \\
\hline 155 & 112 & 246 & 200 & 10,367 & 9,663 & 52,978 & 0 & 0 & 20.1 & 119.8 \\
\hline
\end{tabular}

Table 16.--Yield table for Stand 12, Kaniksu National Forest, Trout Creek Ranger District, Haines Point

\begin{tabular}{|c|c|c|c|c|c|c|c|c|c|c|}
\hline \multirow[b]{2}{*}{ Age } & \multirow[b]{2}{*}{ Trees/acre } & \multirow[b]{2}{*}{$\mathrm{BA} / \mathrm{acre}$} & \multirow[b]{2}{*}{$\mathrm{CCF}$} & \multicolumn{3}{|c|}{ Volume per acre } & \multirow[b]{2}{*}{ Accretion } & \multirow[b]{2}{*}{ Mortality } & \multirow[b]{2}{*}{$\bar{x}$ d.b.h. } & \multirow[b]{2}{*}{$\bar{x}$ height } \\
\hline & & & & $\begin{array}{l}\text { Total } \\
\mathrm{ft}^{3}\end{array}$ & $\begin{array}{l}\text { Merchantable } \\
\mathrm{ft}^{3}\end{array}$ & $\begin{array}{l}\text { Scribner } \\
\text { bd.ft. }\end{array}$ & & & & \\
\hline & & $F t^{2}$ & & & & & $F t^{3}$ & $F t^{3}$ & Inches & Feet \\
\hline 60 & 286 & 48 & 78 & 1,013 & 565 & 604 & 145 & 11 & 5.6 & 40.3 \\
\hline 70 & 254 & 87 & 112 & 2,352 & 1,991 & 3,741 & 148 & 18 & 7.9 & 55.4 \\
\hline 80 & 234 & 117 & 134 & 3,652 & 3,164 & 12,855 & 165 & 24 & 9.6 & 64.7 \\
\hline 90 & 219 & 145 & 151 & 5,057 & 4,343 & 19,983 & 193 & 35 & 11.0 & 73.2 \\
\hline 100 & 205 & 173 & 168 & 6,637 & 5,663 & 27,633 & 212 & 60 & 12.4 & 81.1 \\
\hline 110 & 190 & 196 & 179 & 8,159 & 6,944 & 34,889 & 209 & 107 & 13.8 & 88.4 \\
\hline 120 & 171 & 206 & 183 & 9,182 & 7,767 & 39,640 & 198 & 165 & 14.9 & 95.3 \\
\hline 130 & 149 & 200 & 175 & 9,503 & 8,013 & 41,343 & 169 & 215 & 15.7 & 101.8 \\
\hline 140 & 125 & 181 & 157 & 9,039 & 7,618 & 39,586 & 166 & 223 & 16.3 & 108.0 \\
\hline 150 & 103 & 162 & 144 & 8,464 & 7,080 & 36,909 & 129 & 258 & 17.0 & 114.2 \\
\hline 160 & 81 & 133 & 121 & 7,175 & 6,008 & 31,483 & 0 & 0 & 17.3 & 120.3 \\
\hline
\end{tabular}


Table 17.--Yield table for Stand 13, Kaniksu National Forest, Noxon Ranger District, Squaw Peak

\begin{tabular}{|c|c|c|c|c|c|c|c|c|c|c|}
\hline \multirow[b]{2}{*}{ Age } & \multirow[b]{2}{*}{ Trees/acre } & \multirow[b]{2}{*}{$\mathrm{BA} / \mathrm{acre}$} & \multirow[b]{2}{*}{$\mathrm{CCF}$} & \multicolumn{3}{|c|}{ Volume per acre } & \multirow[b]{2}{*}{ Accretion } & \multirow[b]{2}{*}{ Mortality } & \multirow[b]{2}{*}{$\overline{\mathrm{x}} \mathrm{d} . \mathrm{b} . \mathrm{h}}$. & \multirow[b]{2}{*}{$\bar{x}$ height } \\
\hline & & & & $\begin{array}{l}\text { Total } \\
\mathrm{ft}^{3}\end{array}$ & $\begin{array}{c}\text { Merchantable } \\
\mathrm{ft}^{3}\end{array}$ & $\begin{array}{c}\text { Scribner } \\
\text { bd.ft. }\end{array}$ & & & & \\
\hline & & $F t^{2}$ & & & & & $F t^{3}$ & $F t^{3}$ & Inches & Feet \\
\hline 85 & 91 & 91 & 99 & 3,681 & 3,402 & 18,079 & 67 & 18 & 13.5 & 65.5 \\
\hline 95 & 86 & 100 & 104 & 4,168 & 3,865 & 20,901 & 68 & 21 & 14.6 & 71.9 \\
\hline 105 & 81 & 109 & 108 & 4,636 & 4,297 & 23,677 & 66 & 23 & 15.6 & 78.2 \\
\hline 115 & 77 & 116 & 112 & 5,062 & 4,677 & 26,099 & 71 & 26 & 16.6 & 84.5 \\
\hline 125 & 73 & 124 & 117 & 5,513 & 5.064 & 28,589 & 73 & 28 & 17.6 & 90.9 \\
\hline 135 & 70 & 132 & 120 & 5,959 & 5,484 & 31,073 & 73 & 31 & 18.6 & 96.6 \\
\hline 145 & 66 & 138 & 122 & 6,378 & 5,873 & 33,414 & 70 & 34 & 19.5 & 101.9 \\
\hline 155 & 63 & 143 & 124 & 6,743 & 6,214 & 35,390 & 73 & 36 & 20.4 & 106.9 \\
\hline 165 & 60 & 148 & 125 & 7,114 & 6,559 & 37,482 & 73 & 39 & $21 \cdot 2$ & 111.4 \\
\hline 175 & 57 & 153 & 127 & 7,455 & 6,879 & 39,494 & 73 & 41 & 22.1 & 115.6 \\
\hline 185 & 55 & 157 & 128 & 7,770 & 7,175 & 41,339 & 0 & 0 & 22.9 & 119.5 \\
\hline
\end{tabular}

Table 18.--Yield table for Stand 14, Kootenai National Forest, Libby Ranger District, Granite Creek

Volume per acre

Total Merchantable Scribner

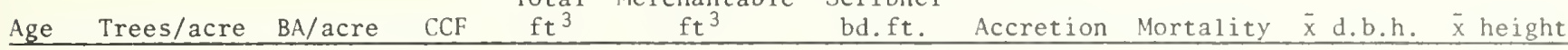

\begin{tabular}{|c|c|c|c|c|c|c|c|c|c|c|}
\hline & & $F t^{2}$ & & & & & $F t^{3}$ & $F t^{3}$ & Inches & Feet \\
\hline 37 & 113 & 45 & 54 & 872 & 599 & 1,180 & 80 & 5 & 8.5 & 38.7 \\
\hline 47 & 105 & 63 & 69 & 1,625 & 1,219 & 3,629 & 92 & 8 & 10.5 & 54.3 \\
\hline 57 & 99 & 80 & 81 & 2,464 & 1,877 & 7,263 & 99 & 12 & 12.1 & 65.0 \\
\hline 67 & 94 & 94 & 91 & 3,335 & 2,531 & 10,901 & 112 & 16 & 13.5 & 74.4 \\
\hline 77 & 90 & 109 & 100 & 4,287 & 3,217 & 14,724 & 118 & 22 & 14.9 & 82.8 \\
\hline 87 & 85 & 122 & 107 & 5,255 & 3,913 & 18,528 & 127 & 26 & 16.2 & 90.4 \\
\hline 97 & 81 & 136 & 115 & 6,266 & 4,638 & 22,396 & 130 & 31 & 17.5 & 97.2 \\
\hline 107 & 77 & 149 & 121 & 7,260 & 5,355 & 26,561 & 129 & 36 & 18.8 & 103.5 \\
\hline 117 & 73 & 160 & 127 & 8,198 & 6,039 & 30,674 & 127 & 44 & 20.1 & 109.4 \\
\hline 127 & 68 & 168 & 130 & 9,035 & 6,658 & 34,509 & 129 & 55 & 21.3 & 114.9 \\
\hline 137 & 63 & 175 & 132 & 9,784 & 7,231 & 38,443 & 0 & 0 & 22.6 & 120.4 \\
\hline
\end{tabular}

Table 19.--Yield table for Stand 15, Kootenai National Forest,

Rexford Ranger District, Alkali Lake

\begin{tabular}{|c|c|c|c|c|c|c|c|c|c|c|c|}
\hline \multirow[b]{2}{*}{ Age } & \multirow[b]{2}{*}{ Trees/acre } & \multirow[b]{2}{*}{$\mathrm{BA} / \mathrm{acre}$} & \multirow[b]{2}{*}{$\mathrm{CCF}$} & \multicolumn{3}{|c|}{ Volume per acre } & \multirow[b]{2}{*}{ Accretion } & \multirow[b]{2}{*}{ Mortality } & \multirow[b]{2}{*}{$\bar{x} \mathrm{~d}, \mathrm{~b}, \mathrm{~h}$. } & \multirow[b]{2}{*}{$\bar{x}$} & \multirow[b]{2}{*}{ height } \\
\hline & & & & $\begin{array}{c}\text { Total } \\
\mathrm{ft}^{3}\end{array}$ & $\begin{array}{c}\text { Merchantable } \\
\mathrm{ft}^{3} \\
\end{array}$ & $\begin{array}{c}\text { Scribner } \\
\text { bd.ft. }\end{array}$ & & & & & \\
\hline & & $F t^{2}$ & & & & & $F t^{3}$ & $F t^{3}$ & Inches & & Feet \\
\hline 24 & 55 & 16 & 20 & 198 & 129 & 0 & 60 & 2 & 7.3 & & 31.1 \\
\hline 34 & 50 & 32 & 34 & 782 & 586 & 1,741 & 85 & 3 & 10.8 & & 56.4 \\
\hline 44 & 48 & 48 & 47 & 1,597 & 1,207 & 5,214 & 95 & 6 & 13.5 & & 73.0 \\
\hline 54 & 46 & 62 & 58 & 2,484 & 1,865 & 8,715 & 104 & 10 & 15.7 & & 85.5 \\
\hline 64 & 44 & 75 & 67 & 3,421 & 2,560 & 12,337 & 108 & 14 & 17.7 & & 95.6 \\
\hline 74 & 42 & 87 & 75 & 4,356 & 3,275 & 16,771 & 132 & 18 & 19.5 & & 104.0 \\
\hline 84 & 40 & 102 & 84 & 5,496 & 4,150 & 22,519 & 123 & 23 & 21.6 & & 111.2 \\
\hline 94 & 38 & 114 & 91 & 6,494 & 4,917 & 27,947 & 126 & 28 & 23.4 & & 117.4 \\
\hline 104 & 37 & 126 & 97 & 7,482 & 5,671 & 33,431 & 135 & 32 & 25.1 & & 122.9 \\
\hline 114 & 35 & 137 & 103 & 8,511 & 6,447 & 39,329 & 126 & 37 & 26.8 & & 127.7 \\
\hline 124 & 33 & 147 & 107 & 9,406 & 7.114 & 44,620 & 0 & 0 & 28.4 & & 132.1 \\
\hline
\end{tabular}


Table 20.--Yield table for Stand 16, Kootenai National Forest, Troy Ranger District, Cheer Creek

Volume per acre

Total Merchantable Scribner

Age Trees/acre BA/acre CCF $\mathrm{ft}^{3} \quad \mathrm{ft}^{3}$ bd.ft. Accretion Mortality $\bar{x} \mathrm{~d} . \mathrm{b} . \mathrm{h} . \overline{\mathrm{x}}$ height

\begin{tabular}{|c|c|c|c|c|c|c|c|c|c|c|}
\hline & & $F t^{2}$ & & & & & $F t^{3}$ & $F t^{3}$ & Inches & Feet \\
\hline 25 & 200 & 11 & 34 & 117 & 0 & 0 & 18 & 2 & 3.2 & 20.9 \\
\hline 35 & 167 & 20 & 46 & 273 & 56 & 0 & 26 & 3 & 4.6 & 29.9 \\
\hline 45 & 146 & 30 & 57 & 498 & 353 & 0 & 32 & 5 & 6.1 & 38.5 \\
\hline 55 & 131 & 38 & 66 & 762 & 689 & 137 & 38 & 7 & 7.3 & 46.6 \\
\hline 65 & 119 & 46 & 73 & 1,071 & 1,032 & 1,110 & 48 & 9 & 8.4 & 54.6 \\
\hline 75 & 109 & 56 & 81 & 1,455 & 1,391 & 2,654 & 51 & 14 & 9.7 & 62.4 \\
\hline 85 & 99 & 63 & 87 & 1,823 & 1,705 & 3,813 & 61 & 19 & 10.8 & 69.8 \\
\hline 95 & 90 & 71 & 93 & 2,247 & 2,040 & 4,937 & 60 & 24 & 12.0 & 76.9 \\
\hline 105 & 81 & 76 & 97 & 2,609 & 2,299 & 5,650 & 61 & 27 & 13.1 & 83.5 \\
\hline 115 & 74 & 81 & 100 & 2,956 & 2,529 & 6,159 & 66 & 30 & 14.2 & 89.8 \\
\hline 125 & 67 & 86 & 103 & 3,321 & 2,763 & 6,544 & 0 & 0 & 15.3 & 95.7 \\
\hline
\end{tabular}

Table 21.--Yield table for Stand 17, Kootenai National Forest, Warland Ranger District, Cripple Horse

\begin{tabular}{|c|c|c|c|c|c|c|c|c|c|c|}
\hline \multirow[b]{2}{*}{ Age } & \multirow[b]{2}{*}{ Trees/acre } & \multirow[b]{2}{*}{$\mathrm{BA} /$ acre } & \multirow[b]{2}{*}{$\mathrm{CCF}$} & \multicolumn{3}{|c|}{ Volume per acre } & \multirow[b]{2}{*}{ Accretion } & \multirow[b]{2}{*}{ Mortality } & \multirow[b]{2}{*}{$\bar{x}$ d.b.h. } & \multirow[b]{2}{*}{$\bar{x}$ height } \\
\hline & & & & $\begin{array}{l}\text { Total } \\
\mathrm{ft}^{3}\end{array}$ & $\begin{array}{l}\text { Merchantable } \\
\mathrm{ft}^{3}\end{array}$ & $\begin{array}{c}\text { Soribner } \\
\text { bd.ft. }\end{array}$ & & & & \\
\hline & & $F t^{2}$ & & & & & $F t^{3}$ & $F t^{3}$ & Inches & Feet \\
\hline 35 & 159 & 50 & 63 & 776 & 497 & 676 & 130 & 5 & 7.6 & 32.3 \\
\hline 45 & 146 & 86 & 90 & 2,023 & 1,466 & 3,549 & 161 & 9 & 10.4 & 53.9 \\
\hline 55 & 139 & 118 & 110 & 3,540 & 2,626 & 9,932 & 173 & 14 & 12.5 & 68.6 \\
\hline 65 & 133 & 145 & 126 & 5,130 & 3,824 & 16,946 & 191 & 20 & 14.2 & 79.6 \\
\hline 75 & 128 & 173 & 140 & 6,839 & 5,095 & 24,106 & 193 & 27 & 15.7 & 88.6 \\
\hline 85 & 123 & 197 & 151 & 8,492 & 6,321 & 31,001 & 203 & 34 & 17.1 & 95.8 \\
\hline 95 & 118 & 220 & 162 & 10,179 & 7,577 & 38,213 & 196 & 41 & 18.5 & 101.9 \\
\hline 105 & 113 & 241 & 170 & 11,727 & 8,740 & 45,295 & 192 & 48 & 19.8 & 107.1 \\
\hline 115 & 108 & 258 & 178 & 13,167 & 9,829 & 52,347 & 187 & 54 & 20.9 & 111.6 \\
\hline 125 & 104 & 274 & 185 & 14,495 & 10,829 & 58,946 & 175 & 60 & 22.0 & 115.6 \\
\hline 135 & 100 & 285 & 191 & 15,643 & 11,698 & 64,866 & 0 & 0 & 22.9 & 119.2 \\
\hline
\end{tabular}

Table 22.--Yield table for Stand 18, Lewis and Clark National Forest, White Sulphur springs Ranger District, Deadman \#3

\begin{tabular}{|c|c|c|c|c|c|c|c|c|c|c|}
\hline \multirow[b]{2}{*}{ Age } & \multirow[b]{2}{*}{ Trees/acre } & \multirow[b]{2}{*}{$\mathrm{BA} /$ acre } & \multirow[b]{2}{*}{$\mathrm{CCF}$} & \multicolumn{3}{|c|}{ Volume per acre } & \multirow[b]{2}{*}{ Accretion } & \multirow[b]{2}{*}{ Mortality } & \multirow[b]{2}{*}{$\bar{x}$ d.b.h. } & \multirow[b]{2}{*}{$\bar{x}$ height } \\
\hline & & & & $\begin{array}{c}\text { Total } \\
\mathrm{ft}^{3} \\
\end{array}$ & $\begin{array}{c}\text { Merchantable } \\
\mathrm{ft}^{3}\end{array}$ & $\begin{array}{c}\text { Scribner } \\
\text { bd.ft. }\end{array}$ & & & & \\
\hline & & $F t^{2}$ & & & & & $F t^{3}$ & $F t^{3}$ & Inches & Feet \\
\hline 24 & 380 & 22 & 49 & 182 & 0 & 0 & 55 & 3 & 3.2 & 15.6 \\
\hline 34 & 318 & 45 & 76 & 702 & 272 & 0 & 56 & 8 & 5.1 & 29.8 \\
\hline 44 & 281 & 61 & 90 & 1,177 & 879 & 0 & 42 & 12 & 6.3 & 37.6 \\
\hline 54 & 254 & 68 & 95 & 1,481 & 1,237 & 458 & 47 & 13 & 7.0 & 42.4 \\
\hline 64 & 231 & 76 & 100 & 1,822 & 1,591 & 3,124 & 52 & 15 & 7.8 & 46.9 \\
\hline 74 & 212 & 85 & 105 & 2,198 & 1,951 & 6,737 & 55 & 16 & 8.6 & 51.1 \\
\hline 84 & 197 & 93 & 110 & 2,586 & 2,295 & 9,627 & 59 & 16 & 9.3 & 55.1 \\
\hline 94 & 184 & 102 & 116 & 3,007 & 2,642 & 11,862 & 65 & 18 & 10.1 & 58.9 \\
\hline 104 & 173 & 111 & 122 & 3,478 & 3,026 & 14,108 & 65 & 21 & 10.9 & 62.4 \\
\hline 114 & 163 & 120 & 127 & 3,921 & 3,388 & 16,269 & 64 & 25 & 11.6 & 65.7 \\
\hline 124 & 153 & 127 & 130 & 4,319 & 3,716 & 18,198 & 0 & 0 & 12.3 & 68.8 \\
\hline
\end{tabular}


Table 23.--Yield table for Stand 19, Lewis and Clark National Forest, White Sulphur Springs Ranger District, Deadman \#2

\begin{tabular}{|c|c|c|c|c|c|c|c|c|c|c|}
\hline \multirow{3}{*}{ Age } & \multirow[b]{2}{*}{ Trees/acre } & \multirow[b]{2}{*}{ BA/acre } & \multirow[b]{2}{*}{ CCF } & \multicolumn{3}{|c|}{ Volume per acre } & \multirow[b]{2}{*}{ Accretion } & \multirow[b]{2}{*}{ Mortality } & \multirow[b]{2}{*}{$\bar{x}$ d.b.h. } & \multirow[b]{2}{*}{$\bar{x}$ height } \\
\hline & & & & $\begin{array}{c}\text { Total } \\
\mathrm{ft}^{3} \\
\end{array}$ & $\begin{array}{c}\text { Merchantable } \\
\mathrm{ft}^{3}\end{array}$ & $\begin{array}{c}\text { Scribner } \\
\text { bd.ft. }\end{array}$ & & & & \\
\hline & & $F t^{2}$ & & & & & $F t^{3}$ & $F t^{3}$ & Inches & Feet \\
\hline 24 & 340 & 16 & 40 & 131 & 0 & 0 & 46 & 2 & 2.9 & 14.8 \\
\hline 34 & 281 & 37 & 65 & 567 & 277 & 0 & 59 & 7 & 4.9 & 29.1 \\
\hline 44 & 248 & 54 & 82 & 1,087 & 975 & 103 & 41 & 11 & 6.3 & 38.8 \\
\hline 54 & 223 & 62 & 88 & 1,387 & 1,374 & 753 & 46 & 12 & 7.1 & 43.8 \\
\hline 64 & 204 & 70 & 93 & 1,720 & 1,772 & 3,607 & 53 & 14 & 7.9 & 48.5 \\
\hline 74 & 187 & 79 & 100 & 2,111 & 2,117 & 6,674 & 53 & 15 & 8.8 & 52.9 \\
\hline 84 & 174 & 88 & 105 & 2,497 & 2,438 & 9,301 & 59 & 15 & 9.6 & 57.0 \\
\hline 94 & 163 & 97 & 111 & 2,934 & 2,830 & 11,736 & 62 & 17 & 10.4 & 60.9 \\
\hline 104 & 154 & 106 & 117 & 3,385 & 2,993 & 13,936 & 68 & 21 & 11.3 & 64.5 \\
\hline 114 & 145 & 116 & 123 & 3,859 & 3,343 & 16,225 & 65 & 26 & 12.1 & 67.9 \\
\hline 124 & 136 & 123 & 126 & 4,251 & 3,670 & 18,154 & 0 & 0 & 12.9 & 71.0 \\
\hline
\end{tabular}

Table 24.--Yield table for stand 20, Lewis and Clark National Forest, White Sulphur Springs Ranger District, Deadman \#I

\begin{tabular}{|c|c|c|c|c|c|c|c|c|c|c|}
\hline \multirow[b]{2}{*}{ Age } & \multirow[b]{2}{*}{ Trees/acre } & \multirow[b]{2}{*}{ BA/acre } & \multirow[b]{2}{*}{ CCF } & \multicolumn{3}{|c|}{ Volume per acre } & \multirow[b]{2}{*}{ Accretion } & \multirow[b]{2}{*}{ 1ortality } & \multirow[b]{2}{*}{$\bar{x}$ d.b.h. } & \multirow[b]{2}{*}{$\bar{x}$ height } \\
\hline & & & & $\begin{array}{c}\begin{array}{c}\text { Total } \\
\mathrm{ft}^{3}\end{array} \\
\end{array}$ & $\begin{array}{c}\text { Merchantable } \\
\mathrm{ft}^{3}\end{array}$ & $\begin{array}{c}\text { Scribner } \\
\text { bd.ft. }\end{array}$ & & & & \\
\hline & & $F t^{2}$ & & & & & $F t^{3}$ & $F t^{3}$ & Inches & Feet \\
\hline 23 & 300 & 12 & 34 & 97 & 0 & 0 & 31 & 2 & 2.8 & 13.1 \\
\hline 33 & 245 & 27 & 50 & 386 & 140 & 0 & 50 & 5 & 4.5 & 26.0 \\
\hline 43 & 213 & 43 & 67 & 834 & 578 & 83 & 41 & 8 & 6.1 & 37.6 \\
\hline 53 & 191 & 52 & 75 & 1,159 & 963 & 513 & 43 & 10 & 7.1 & 43.7 \\
\hline 63 & 174 & 60 & 82 & 1,487 & 1,303 & 2,781 & 47 & 12 & 8.0 & 48.7 \\
\hline 73 & 160 & 69 & 87 & 1,835 & 1,627 & 6,251 & 51 & 13 & 8.9 & 53.2 \\
\hline 83 & 149 & 77 & 94 & 2,223 & 1,960 & 8,358 & 53 & 14 & 9.7 & 57.5 \\
\hline 93 & 140 & 86 & 99 & 2,621 & 2,288 & 10,391 & 61 & 15 & 10.6 & 61.5 \\
\hline 103 & 132 & 95 & 106 & 3,072 & 2,657 & 12,678 & 63 & 19 & 11.5 & 65.2 \\
\hline 113 & 124 & 104 & 111 & 3,508 & 3,020 & 14,798 & 67 & 24 & 12.4 & 68.7 \\
\hline 123 & 115 & 111 & 116 & 3,936 & 3,379 & 16,872 & 0 & 0 & 13.3 & 72.0 \\
\hline
\end{tabular}

Table 25.--Yield table for Stand 21, Lolo National Forest, Missoula Ranger District, Teepee Creek

\begin{tabular}{|c|c|c|c|c|c|c|c|c|c|c|}
\hline \multirow[b]{2}{*}{ Age } & \multirow[b]{2}{*}{ Trees/acre } & \multirow[b]{2}{*}{$\mathrm{BA} / \mathrm{acre}$} & \multirow[b]{2}{*}{ CCF } & \multicolumn{3}{|c|}{ Volume per acre } & \multirow[b]{2}{*}{ Accretion } & \multirow[b]{2}{*}{ Mortality } & \multirow[b]{2}{*}{$\bar{x}$ d.b.h. } & \multirow[b]{2}{*}{$\bar{x}$ height } \\
\hline & & & & $\begin{array}{c}\text { Total } \\
\mathrm{ft}^{3} \\
\end{array}$ & $\begin{array}{c}\text { Merchantable } \\
\mathrm{ft}^{3}\end{array}$ & $\begin{array}{l}\text { Scribner } \\
\text { bd.ft. }\end{array}$ & & & & \\
\hline & & $F t^{2}$ & & & & & $F t^{3}$ & $F t^{3}$ & Inches & Feet \\
\hline 49 & 979 & 106 & 188 & 1,676 & 1,012 & 1,497 & 113 & 15 & 4.4 & 22.2 \\
\hline 59 & 811 & 146 & 204 & 2,657 & 1,747 & 3,220 & 127 & 31 & 5.7 & 29.8 \\
\hline 69 & 657 & 173 & 210 & 3,618 & 2,511 & 5,675 & 117 & 51 & 7.0 & 37.8 \\
\hline 79 & 525 & 182 & 199 & 4,275 & 3,145 & 8,490 & 115 & 65 & 8.0 & 45.5 \\
\hline 89 & 423 & 182 & 186 & 4,775 & 3,644 & 11,272 & 114 & 73 & 8.9 & 52.2 \\
\hline 99 & 346 & 181 & 174 & 5,185 & 4,056 & 14,008 & 105 & 77 & 9.8 & 58.2 \\
\hline 109 & 288 & 177 & 162 & 5,460 & 4,334 & 16,420 & 102 & 78 & 10.6 & 63.5 \\
\hline 119 & 244 & 172 & 155 & 5,702 & 4,573 & 18,584 & 90 & 77 & 11.4 & 68.1 \\
\hline 129 & 208 & 165 & 147 & 5,825 & 4,710 & 20,206 & 89 & 74 & 12.1 & 72.4 \\
\hline 139 & 179 & 161 & 141 & 5,971 & 4,849 & 21,691 & 82 & 72 & 12.8 & 76.5 \\
\hline 149 & 156 & 156 & 134 & 6,068 & 4,946 & 22,916 & 0 & 0 & 13.5 & 80.3 \\
\hline
\end{tabular}


Table 26.--Yield table for stand 22, Beaverhead National Forest, Dillon Ranger District, Dinner Station

\begin{tabular}{|c|c|c|c|c|c|c|c|c|c|c|}
\hline \multirow[b]{2}{*}{ Age } & \multirow[b]{2}{*}{ Trees/acre } & \multirow[b]{2}{*}{$\mathrm{BA} / \mathrm{acre}$} & \multirow[b]{2}{*}{$\mathrm{CCF}$} & \multicolumn{3}{|c|}{ Volume per acre } & \multirow[b]{2}{*}{ Accretion } & \multirow[b]{2}{*}{ Mortality } & \multirow[b]{2}{*}{$\bar{x}$ d.b.h. } & \multirow[b]{2}{*}{$\bar{x}$ height } \\
\hline & & & & $\begin{array}{l}\text { Total } \\
\mathrm{ft}^{3}\end{array}$ & $\begin{array}{c}\text { Merchantable } \\
\mathrm{ft}^{3}\end{array}$ & $\begin{array}{c}\text { Scribner } \\
\text { bd.ft. }\end{array}$ & & & & \\
\hline & & $F t^{2}$ & & & & & $F t^{3}$ & $F t^{3}$ & Inches & Feet \\
\hline 45 & 370 & 46 & 78 & 600 & 191 & 103 & 141 & 7 & 4.8 & 24.3 \\
\hline 55 & 325 & 98 & 126 & 1,934 & 1,604 & 3,596 & 201 & 15 & 7.4 & 38.0 \\
\hline 65 & 297 & 156 & 169 & 3,789 & 3,357 & 13,370 & 241 & 26 & 9.8 & 47.8 \\
\hline 75 & 278 & 210 & 201 & 5,946 & 5,216 & 24,470 & 290 & 49 & 11.8 & 56.2 \\
\hline 85 & 258 & 262 & 238 & 8,350 & 7,248 & 35,982 & 300 & 111 & 13.6 & 63.5 \\
\hline 95 & 233 & 294 & 259 & 10,243 & 8,793 & 44,573 & 272 & 223 & 15.2 & 69.8 \\
\hline 105 & 199 & 289 & 250 & 10,733 & 9,117 & 46,605 & 255 & 300 & 16.3 & 74.9 \\
\hline 115 & 162 & 262 & 224 & 10,279 & 8,711 & 45,009 & 237 & 340 & 17.2 & 79.5 \\
\hline 125 & 125 & 225 & 190 & 9,250 & 7,775 & 40,275 & 186 & 346 & 18.2 & 83.8 \\
\hline 135 & 89 & 178 & 149 & 7,645 & 6,362 & 33,047 & 125 & 355 & 19.1 & 88.0 \\
\hline 145 & 58 & 120 & 107 & 5,347 & 4,444 & 23,175 & 0 & 0 & 19.5 & 91.5 \\
\hline
\end{tabular}

Table 27.--Yield table for Stand 23, Beaverhead National Forest, Wisdom Ranger District, Tie Creek Burn

\begin{tabular}{|c|c|c|c|c|c|c|c|c|c|c|}
\hline \multirow[b]{2}{*}{ Age } & \multirow[b]{2}{*}{ Trees/acre } & \multirow[b]{2}{*}{$\mathrm{BA} / \mathrm{acre}$} & \multirow[b]{2}{*}{$\mathrm{CCF}$} & \multicolumn{3}{|c|}{ Volume per acre } & \multirow[b]{2}{*}{ Accretion } & \multirow[b]{2}{*}{ Mortality } & \multirow[b]{2}{*}{$\bar{x}$ d.b.h. } & \multirow[b]{2}{*}{$\vec{x}$ height } \\
\hline & & & & $\begin{array}{c}\text { Total } \\
\mathrm{ft}^{3}\end{array}$ & $\begin{array}{c}\text { Merchantable } \\
\mathrm{ft}^{3}\end{array}$ & $\begin{array}{c}\text { Scribner } \\
\text { bd.ft. }\end{array}$ & & & & \\
\hline & & $F t^{2}$ & & & & & $F t^{3}$ & $F t^{3}$ & Inches & Feet \\
\hline 17 & 280 & 5 & 29 & 35 & 0 & 0 & 17 & 1 & 1.9 & 9.5 \\
\hline 27 & 211 & 14 & 34 & 197 & 14 & 0 & 37 & 3 & 3.5 & 22.2 \\
\hline 37 & 176 & 27 & 47 & 531 & 326 & 0 & 46 & 6 & 5.3 & 35.8 \\
\hline 47 & 156 & 39 & 59 & 927 & 746 & 264 & 37 & 9 & 6.8 & 45.8 \\
\hline 57 & 142 & 47 & 66 & 1,212 & 1,053 & 1,879 & 42 & 10 & 7.8 & 50.6 \\
\hline 67 & 130 & 55 & 73 & 1,539 & 1,364 & 4,987 & 52 & 11 & 8.8 & 55.2 \\
\hline 77 & 121 & 65 & 80 & 1,948 & 1,712 & 7,513 & 53 & 12 & 9.9 & 59.7 \\
\hline 87 & 114 & 74 & 87 & 2,359 & 2,047 & 9,607 & 59 & 14 & 10.9 & 64.0 \\
\hline 97 & 107 & 83 & 93 & 2,807 & 2,417 & 11,732 & 62 & 19 & 11.9 & 68.1 \\
\hline 107 & 100 & 91 & 99 & 3,243 & 2,780 & 13,813 & 68 & 25 & 12.9 & 72.1 \\
\hline 117 & 93 & 98 & 103 & 3,676 & 3,145 & 15,906 & 0 & 0 & 13.9 & 75.8 \\
\hline
\end{tabular}

Table 28.--Yield table for Stand 24, Flathead National Forest, Glacier View Ranger District, Camas Creek

\begin{tabular}{|c|c|c|c|c|c|c|c|c|c|c|}
\hline \multirow[b]{2}{*}{ Age } & \multirow[b]{2}{*}{ Trees/acre } & \multirow[b]{2}{*}{$\mathrm{BA} / \mathrm{acre}$} & \multirow[b]{2}{*}{$\mathrm{CCF}$} & \multicolumn{3}{|c|}{ Volume per acre } & \multirow[b]{2}{*}{ Accretion } & \multirow[b]{2}{*}{ Mortality } & \multirow[b]{2}{*}{$\bar{x}$ d.b.h. } & \multirow[b]{2}{*}{$\bar{x}$ height } \\
\hline & & & & $\begin{array}{l}\text { Total } \\
\mathrm{ft}^{3} \\
\end{array}$ & $\begin{array}{c}\text { Merchantable } \\
\mathrm{ft}^{3}\end{array}$ & $\begin{array}{c}\text { Scribner } \\
\text { bd.ft. }\end{array}$ & & & & \\
\hline & & $F t^{2}$ & & & & & $F t^{3}$ & $F t^{3}$ & Inches & Feet \\
\hline 65 & 199 & 34 & 51 & 722 & 385 & 0 & 49 & 8 & 5.6 & 39.4 \\
\hline 75 & 177 & 45 & 67 & 1,130 & 829 & 424 & 36 & 10 & 6.8 & 47.8 \\
\hline 85 & 161 & 51 & 72 & 1,386 & 1,071 & 1,974 & 37 & 11 & 7.6 & 52.2 \\
\hline 95 & 148 & 56 & 76 & 1,640 & 1,281 & 3,535 & 41 & 12 & 8.4 & 56.5 \\
\hline 105 & 137 & 62 & 80 & 1,930 & 1,493 & 4,876 & 43 & 13 & 9.1 & 60.6 \\
\hline 115 & 128 & 68 & 84 & 2,232 & 1,696 & 6,182 & 47 & 14 & 9.9 & 64.6 \\
\hline 125 & 120 & 74 & 88 & 2,565 & 1,908 & 7,341 & 51 & 16 & 10.6 & 68.5 \\
\hline 135 & 112 & 80 & 92 & 2,919 & 2,127 & 8,339 & 50 & 20 & 11.4 & 72.2 \\
\hline 145 & 105 & 84 & 94 & 3,221 & 2,301 & 9,215 & 51 & 24 & 12.1 & 75.8 \\
\hline 155 & 98 & 87 & 96 & 3,494 & 2,449 & 9,776 & 54 & 30 & 12.8 & 79.2 \\
\hline 165 & 91 & 90 & 97 & 3,733 & 2,566 & 10,125 & 0 & 0 & 13.5 & 82.5 \\
\hline
\end{tabular}


Table 29.--Yield table for Stand 25, Flathead National Forest,

\begin{tabular}{|c|c|c|c|c|c|c|c|c|c|c|}
\hline \multirow[b]{2}{*}{ Age } & \multirow[b]{2}{*}{ Trees/acre } & \multirow[b]{2}{*}{ BA/acre } & \multicolumn{4}{|c|}{ Volume per acre } & \multirow[b]{2}{*}{ Accretion } & \multirow[b]{2}{*}{ Mortality } & \multirow[b]{2}{*}{$\bar{x}$ d.b.h. } & \multirow[b]{2}{*}{$\bar{x}$ height } \\
\hline & & & $\mathrm{CCF}$ & $\begin{array}{l}\text { Total } \\
\mathrm{ft}^{3}\end{array}$ & $\begin{array}{c}\text { Merchantable } \\
\mathrm{ft}^{3}\end{array}$ & $\begin{array}{c}\text { Scribner } \\
\text { bd.ft. }\end{array}$ & & & & \\
\hline & & $E t^{2}$ & & & & & $F t^{3}$ & $E t^{3}$ & Inches & Feet \\
\hline 48 & 286 & 65 & 93 & 1,418 & 968 & 834 & 138 & 14 & 6.5 & 42.0 \\
\hline 58 & 258 & 101 & 123 & 2,662 & 2,055 & 5,564 & 159 & 19 & 8.5 & 51.8 \\
\hline 68 & 239 & 136 & 147 & 4,062 & 3,074 & 10,803 & 178 & 26 & 10.2 & 59.8 \\
\hline 78 & 224 & 167 & 166 & 5,575 & 4,062 & 15,823 & 196 & 42 & 11.7 & 67.0 \\
\hline 88 & 209 & 195 & 182 & 7,116 & 4,974 & 19,456 & 212 & 73 & 13.1 & 73.7 \\
\hline 98 & 191 & 216 & 194 & 8,505 & 5,709 & 21,819 & 202 & 128 & 14.4 & 79.8 \\
\hline 108 & 170 & 221 & 194 & 9,246 & 6,003 & 22,293 & 186 & 185 & 15.4 & 85.4 \\
\hline 118 & 145 & 209 & 181 & 9,254 & 5,859 & 21,271 & 165 & 215 & 16.3 & 90.5 \\
\hline 128 & 119 & 188 & 161 & 8,749 & 5,419 & 19,197 & 143 & 239 & 17.0 & 95.4 \\
\hline 138 & 95 & 160 & 140 & 7,792 & 4,714 & 16,309 & 122 & 235 & 17.6 & 100.1 \\
\hline 148 & 72 & 131 & 119 & 6,660 & 3,961 & 13,367 & 0 & 0 & 18.2 & 104.5 \\
\hline
\end{tabular}

Table 30.-yield table for Stand 26, Flathead National Forest, Swan Lake Ranger District, Bond Creek

\begin{tabular}{|c|c|c|c|c|c|c|c|c|c|c|}
\hline \multirow{3}{*}{ Age } & \multirow[b]{2}{*}{ Trees/acre } & \multirow[b]{2}{*}{ BA/acre } & \multicolumn{4}{|c|}{ Volume per acre } & \multirow[b]{2}{*}{ Accretion } & \multirow[b]{2}{*}{ Mortality } & \multirow[b]{2}{*}{$\bar{x}$ d.b.h. } & \multirow[b]{2}{*}{$\bar{x}$ height } \\
\hline & & & $\mathrm{CCF}$ & $\begin{array}{c}\text { Total } \\
\mathrm{ft}^{3} \\
\end{array}$ & $\begin{array}{c}\text { Verchantable } \\
\mathrm{ft}^{3}\end{array}$ & $\begin{array}{c}\text { Scribner } \\
\text { bd.ft. }\end{array}$ & & & & \\
\hline & & $F t^{2}$ & & & & & $F t^{3}$ & $F t^{3}$ & Inches & Feet \\
\hline 62 & 75 & 60 & 77 & 1,408 & 1,322 & 4,756 & 199 & 7 & 12.1 & 60.1 \\
\hline 72 & 71 & 114 & 116 & 3,332 & 2,946 & 12,378 & 229 & 17 & 17.1 & 77.6 \\
\hline 82 & 68 & 163 & 145 & 5,446 & 4,444 & 18,662 & 235 & 30 & 21.0 & 90.2 \\
\hline 92 & 64 & 203 & 165 & 7,501 & 5,623 & 22,983 & 270 & 43 & 24.1 & 100.5 \\
\hline 102 & 61 & 245 & 186 & 9,778 & 6,646 & 25,973 & 279 & 58 & $2^{7} .2$ & 109.3 \\
\hline 112 & 57 & 283 & 205 & 11,983 & 7,346 & 27,240 & 281 & 75 & 30.1 & 117.0 \\
\hline 122 & 54 & 317 & 224 & 14,047 & 7,739 & 27,138 & 288 & 91 & 32.8 & 123.8 \\
\hline 132 & 51 & 349 & 242 & 16,016 & 7,821 & 25,922 & 282 & 108 & 35.6 & 129.8 \\
\hline 142 & 47 & 377 & 257 & 17,747 & 7,757 & 24,336 & 254 & 125 & 38.2 & 135.1 \\
\hline 152 & 44 & 397 & 268 & 19,037 & 7,567 & 23,053 & 230 & 139 & 40.5 & 139.8 \\
\hline 162 & 41 & 414 & 277 & 19,943 & 7,465 & 21,514 & 0 & 0 & 12.8 & 144.1 \\
\hline
\end{tabular}

Table 31.--Yield table for stand 27, Gallatin National Forest, Bozeman Ranger District, Face Draw

\begin{tabular}{|c|c|c|c|c|c|c|c|c|c|c|}
\hline \multirow[b]{2}{*}{ Age } & \multirow[b]{2}{*}{ Trees/acre } & \multirow[b]{2}{*}{$\mathrm{BA} / \mathrm{acre}$} & \multirow[b]{2}{*}{$\mathrm{CCF}$} & \multicolumn{3}{|c|}{ Volume per acre } & \multirow[b]{2}{*}{ Accretion } & \multirow[b]{2}{*}{ Mortality } & \multirow[b]{2}{*}{$\bar{x}$ d.b.h. } & \multirow[b]{2}{*}{$\bar{x}$ height } \\
\hline & & & & $\begin{array}{l}\text { Total } \\
\mathrm{ft}^{3}\end{array}$ & $\begin{array}{c}\text { Merchantable } \\
\mathrm{ft}^{3}\end{array}$ & $\begin{array}{l}\text { Scribner } \\
\text { bd.ft. }\end{array}$ & & & & \\
\hline & & $E t^{2}$ & & & & & $F t^{3}$ & $F t^{3}$ & Inches & Feet \\
\hline 25 & 315 & 47 & 73 & 675 & 457 & 700 & 55 & 7 & 5.2 & 26.1 \\
\hline 35 & 279 & 63 & 92 & 1,154 & 876 & 1,044 & 44 & 10 & 6.5 & 35.0 \\
\hline 45 & 252 & 73 & 99 & 1,497 & 1,274 & 1,848 & 47 & 12 & 7.3 & 39.9 \\
\hline 55 & 231 & 82 & 105 & 1,846 & 1,640 & 3,750 & 54 & 14 & 8.1 & 44.6 \\
\hline 65 & 213 & 92 & 111 & 2,252 & 2,029 & 6,653 & 59 & 15 & 8.9 & 49.0 \\
\hline 75 & 198 & 102 & 118 & 2,689 & 2,421 & 9,990 & 64 & 16 & 9.7 & 53.1 \\
\hline 85 & 186 & 113 & 124 & 3,161 & 2,828 & 12,638 & 63 & 19 & 10.5 & 57.0 \\
\hline 95 & 175 & 121 & 129 & 3,607 & 3,192 & 14,746 & 68 & 21 & 11.3 & 60.6 \\
\hline 105 & 164 & 130 & 135 & 4,072 & 3,572 & 16,793 & 66 & 26 & 12.1 & 64.0 \\
\hline 115 & 154 & 137 & 138 & 4,477 & 3,902 & 18,654 & 66 & 32 & 12.8 & 67.2 \\
\hline 125 & 144 & 142 & 140 & 4,826 & 4,187 & 20,228 & 0 & 0 & 13.4 & 70.1 \\
\hline
\end{tabular}


Table 32.--Yield table for Stand 28, Gallatin National Forest, Bozeman Ranger District, Wildhorse

\begin{tabular}{|c|c|c|c|c|c|c|c|c|c|c|}
\hline \multirow[b]{2}{*}{ Age } & \multirow[b]{2}{*}{ Trees/acre } & \multirow[b]{2}{*}{$\mathrm{BA} / \mathrm{acre}$} & \multirow[b]{2}{*}{ CCF } & \multicolumn{3}{|c|}{ Volume per acre } & \multirow[b]{2}{*}{ Accretion } & \multirow[b]{2}{*}{ "lortality } & \multirow[b]{2}{*}{$\bar{x}$ d.b.h. } & \multirow[b]{2}{*}{$\bar{x}$ height } \\
\hline & & & & $\begin{array}{c}\text { Total } \\
\mathrm{ft}^{3}\end{array}$ & $\begin{array}{c}\text { Merchantable } \\
\mathrm{ft}^{3}\end{array}$ & $\begin{array}{l}\text { Scribner } \\
\text { bd.ft. }\end{array}$ & & & & \\
\hline & & $F t^{2}$ & & & & & $F t^{3}$ & $F t^{3}$ & Inches & Feet \\
\hline 21 & 407 & 29 & 63 & 267 & 0 & 0 & 66 & 4 & 3.6 & 17.4 \\
\hline 31 & 346 & 55 & 88 & 883 & 448 & 0 & 56 & 10 & 5.4 & 30.8 \\
\hline 41 & 307 & 70 & 100 & 1,348 & 1,040 & 0 & 48 & 13 & 6.5 & 37.7 \\
\hline 51 & 278 & 78 & 105 & 1,699 & 1,438 & 1,069 & 53 & 15 & 7.2 & 42.4 \\
\hline 61 & 253 & 88 & 111 & 2,084 & 1,832 & 4,018 & 61 & 17 & 8.0 & 46.9 \\
\hline 71 & 233 & 98 & 117 & 2,527 & 2,248 & 7,409 & 63 & 17 & 8.8 & 51.2 \\
\hline 81 & 217 & 107 & 123 & 2,980 & 2,636 & 10,817 & 66 & 18 & 9.5 & 55.2 \\
\hline 91 & 203 & 117 & 129 & 3,459 & 3,033 & 13,777 & 74 & 20 & 10.3 & 58.9 \\
\hline 101 & 191 & 128 & 136 & 3,994 & 3,475 & 16,337 & 74 & 24 & 11.1 & 62.4 \\
\hline 111 & 180 & 137 & 140 & 4,486 & 3,878 & 18,711 & 73 & 29 & 11.8 & 65.7 \\
\hline 121 & 168 & 144 & 144 & 4,918 & 4,234 & 20,817 & 0 & 0 & 12.5 & 68.7 \\
\hline
\end{tabular}

Table 33.--Yield table for stand 29, Kootenai National Forest, Rexford Ranger District, Pinkham Creek

\begin{tabular}{|c|c|c|c|c|c|c|c|c|c|c|}
\hline \multirow[b]{2}{*}{ Age } & \multirow[b]{2}{*}{ Trees/acre } & \multirow[b]{2}{*}{$\mathrm{BA} / \mathrm{acre}$} & \multirow[b]{2}{*}{$\mathrm{CCF}$} & \multicolumn{3}{|c|}{ Volume per acre } & \multirow[b]{2}{*}{ Accretion } & \multirow[b]{2}{*}{ Mortality } & \multirow[b]{2}{*}{$\bar{x}$ d.b.h. } & \multirow[b]{2}{*}{$\bar{x}$ height } \\
\hline & & & & $\begin{array}{l}\text { Total } \\
\mathrm{ft}^{3} \\
\end{array}$ & $\begin{array}{c}\text { Merchantable } \\
\mathrm{ft}^{3}\end{array}$ & $\begin{array}{l}\text { Seribner } \\
\text { bd.ft. }\end{array}$ & & & & \\
\hline & & $F t^{2}$ & & & & & $F t^{3}$ & $F t^{3}$ & Inches & Feet \\
\hline 50 & 208 & 45 & 63 & 960 & 697 & 833 & 166 & 9 & 6.3 & 40.0 \\
\hline 60 & 187 & 94 & 110 & 2,533 & 2,064 & 5,404 & 209 & 17 & 9.6 & 53.8 \\
\hline 70 & 175 & 142 & 147 & 4,455 & 3,319 & 11,723 & 237 & 42 & 12.2 & 63.4 \\
\hline 80 & 162 & 182 & 170 & 6,404 & 4,505 & 16,021 & 266 & 97 & 14.4 & 71.8 \\
\hline 90 & 144 & 210 & 183 & 8,090 & 5,534 & 18,359 & 240 & 195 & 16.3 & 79.2 \\
\hline 100 & 119 & 206 & 175 & 8,547 & 5,784 & 17,478 & 210 & 290 & 17.8 & 85.6 \\
\hline 110 & 89 & 176 & 147 & 7,747 & 5,228 & 13,877 & 152 & 354 & 19.1 & 91.2 \\
\hline 120 & 57 & 125 & 110 & 5,729 & 3,908 & 8,537 & 74 & 346 & 20.0 & 95.7 \\
\hline 130 & 31 & 64 & 66 & 3,007 & 2,089 & 4,797 & 38 & 172 & 19.3 & 98.3 \\
\hline 140 & 19 & 36 & 44 & 1,668 & 1,207 & 3,175 & 30 & 90 & 18.6 & 100.3 \\
\hline 150 & 13 & 24 & 31 & 1,068 & 835 & 2,115 & 0 & 0 & 18.6 & 101.7 \\
\hline
\end{tabular}

Table 34.--Yield table for Stand 30, Lolo National Forest, Ninemile Ranger District, Spring Creek

\begin{tabular}{|c|c|c|c|c|c|c|c|c|c|c|}
\hline \multirow[b]{2}{*}{ Age } & \multirow[b]{2}{*}{ Trees/acre } & \multirow[b]{2}{*}{$\mathrm{BA} / \mathrm{acre}$} & \multirow[b]{2}{*}{ CCF } & \multicolumn{3}{|c|}{ Volume per acre } & \multirow[b]{2}{*}{ Accretion } & \multirow[b]{2}{*}{ "lortality } & \multirow[b]{2}{*}{$\bar{x} d \cdot b \cdot h$. } & \multirow[b]{2}{*}{$\bar{x}$ height } \\
\hline & & & & $\begin{array}{c}\text { Total } \\
\mathrm{ft}^{3} \\
\end{array}$ & $\begin{array}{c}\text { Terchantable } \\
\mathrm{ft}^{3}\end{array}$ & $\begin{array}{c}\text { Scribner } \\
\text { bd.ft. }\end{array}$ & & & & \\
\hline & & $F t^{2}$ & & & & & $F t^{3}$ & $F t^{3}$ & Inches & Feet \\
\hline 205 & 295 & 98 & 136 & 2,448 & 1,956 & 6,397 & 95 & 25 & 7.8 & 47.5 \\
\hline 215 & 248 & 114 & 138 & 3,152 & 2,567 & 9,369 & 96 & 37 & 9.2 & 56.8 \\
\hline 225 & 210 & 124 & 138 & 3,749 & 3,084 & 12,256 & 89 & 47 & 10.4 & 64.7 \\
\hline 235 & 178 & 127 & 134 & 4,169 & 3,476 & 14,755 & 91 & 54 & 11.5 & 71.1 \\
\hline 245 & 152 & 130 & 131 & 4,540 & 3,821 & 17,263 & 85 & 59 & 12.5 & 76.8 \\
\hline 255 & 130 & 128 & 125 & 4,793 & 4,052 & 19,227 & 83 & 62 & 13.5 & 81.8 \\
\hline 265 & 111 & 127 & 120 & 5,003 & 4,239 & 20,885 & 76 & 61 & 14.5 & 86.5 \\
\hline 275 & 96 & 124 & 115 & 5,159 & 4,381 & 22,267 & 71 & 59 & 15.4 & 90.8 \\
\hline 285 & 84 & 122 & 111 & 5,284 & 4,499 & 23,507 & 70 & 57 & 16.3 & 94.7 \\
\hline 295 & 75 & 120 & 108 & 5,412 & 4,617 & 24,737 & 65 & 55 & 17.1 & 98.4 \\
\hline 305 & 66 & 117 & 104 & 5,508 & 4,706 & 25,703 & 0 & 0 & 18.0 & 101.9 \\
\hline
\end{tabular}


Table 35.--Yield table for Stand 31, Colville National Forest, Colville Ranger District, Robins Creek

Volume per acre

Total Merchantable Scribner

Age Trees/acre BA/acre CCF $\mathrm{ft}^{3} \quad \mathrm{ft}^{3} \quad$ bd.ft. Accretion Mortality $\bar{x} \mathrm{~d} . \mathrm{b} . \mathrm{h} . \overline{\mathrm{x}} \mathrm{height}$

\begin{tabular}{|c|c|c|c|c|c|c|c|c|c|c|}
\hline & & Ft & & & & & $F t^{3}$ & $F t^{3}$ & Inches & Feet \\
\hline 60 & 178 & 42 & 76 & 884 & 664 & 2,327 & 88 & 6 & 6.6 & 37.1 \\
\hline 70 & 161 & 69 & 101 & 1,705 & 1,434 & 6,159 & 111 & 10 & 8.8 & 46.2 \\
\hline 80 & 149 & 96 & 122 & 2,721 & 2,464 & 11,266 & 130 & 15 & 10.9 & 55.0 \\
\hline 90 & 139 & 123 & 140 & 3,867 & 3,590 & 17,103 & 159 & 22 & 12.7 & 63.8 \\
\hline 100 & 131 & 153 & 158 & 5,234 & 4,911 & 24,911 & 188 & 30 & 14.6 & 72.2 \\
\hline 110 & 124 & 184 & 177 & 6,814 & 6,413 & 34,352 & 204 & 40 & 16.5 & 80.0 \\
\hline 120 & 117 & 215 & 195 & 8,454 & 7,971 & 43,868 & 225 & 50 & 18.3 & 87.3 \\
\hline 130 & 112 & 246 & 217 & 10,205 & 9,646 & 54,094 & 226 & 62 & 20.1 & 94.0 \\
\hline 140 & 106 & 272 & 234 & 11,842 & 11,219 & 63,653 & 225 & 73 & 21.7 & 100.2 \\
\hline 150 & 102 & 295 & 250 & 13,363 & 12,723 & 72,847 & 240 & 85 & 23.1 & 105.9 \\
\hline 160 & 97 & 318 & 266 & 14,916 & 14,278 & 82,362 & 0 & 0 & 24.5 & 111.2 \\
\hline
\end{tabular}

Table 36.--Yield table for Stand 32, Colville National Forest, Kettle Falls Ranger District, Long Shot Mine

\begin{tabular}{|c|c|c|c|c|c|c|c|c|c|c|}
\hline \multirow[b]{2}{*}{ Age } & \multirow[b]{2}{*}{ Trees/acre } & \multirow[b]{2}{*}{$\mathrm{BA} / \mathrm{acre}$} & \multirow[b]{2}{*}{$\mathrm{CCF}$} & \multicolumn{3}{|c|}{ Volume per acre } & \multirow[b]{2}{*}{ Accretion } & \multirow[b]{2}{*}{ Mortality } & \multirow[b]{2}{*}{$\bar{x}$ d.b.h. } & \multirow[b]{2}{*}{$\bar{x}$ height } \\
\hline & & & & $\begin{array}{c}\text { Total } \\
\mathrm{ft}^{3}\end{array}$ & $\begin{array}{c}\text { Merchantable } \\
\mathrm{ft}^{3} \\
\end{array}$ & $\begin{array}{l}\text { Scribner } \\
\text { bd.ft. }\end{array}$ & & & & \\
\hline & & $F t^{2}$ & & & & & $F t^{3}$ & $F t^{3}$ & Inches & Feet \\
\hline 45 & 122 & 46 & 62 & 1,442 & 1,214 & 3,633 & 77 & 10 & 8.3 & 63.3 \\
\hline 55 & 113 & 62 & 76 & 2,117 & 1,820 & 7,710 & 86 & 13 & 10.0 & 70.4 \\
\hline 65 & 106 & 77 & 89 & 2,843 & 2,438 & 11,577 & 88 & 20 & 11.6 & 76.9 \\
\hline 75 & 99 & 90 & 97 & 3,518 & 3,007 & 14,879 & 98 & 32 & 12.9 & 82.9 \\
\hline 85 & 91 & 101 & 104 & 4,183 & 3,578 & 18,154 & 97 & 52 & 14.2 & 88.7 \\
\hline 95 & 82 & 106 & 107 & 4,631 & 3,951 & 20,309 & 95 & 81 & 15.4 & 94.2 \\
\hline 105 & 72 & 105 & 104 & 4,774 & 4,074 & 21,160 & 95 & 106 & 16.4 & 99.5 \\
\hline 115 & 60 & 99 & 98 & 4,672 & 3,984 & 20,722 & 74 & 122 & 17.4 & 104.7 \\
\hline 125 & 48 & 87 & 87 & 4,190 & 3,587 & 18,772 & 64 & 124 & 18.2 & 109.9 \\
\hline 135 & 38 & 74 & 75 & 3,598 & 3,097 & 16,305 & 48 & 116 & 19.0 & 115.2 \\
\hline 145 & 28 & 60 & 63 & 2,915 & 2,544 & 13,363 & 0 & 0 & 19.7 & 120.8 \\
\hline
\end{tabular}

Table 37.--Yield table for Stand 33, Colville National Forest Colville Ranger District, Box Canyon

\begin{tabular}{|c|c|c|c|c|c|c|c|c|c|c|}
\hline \multirow[b]{2}{*}{ Age } & \multirow[b]{2}{*}{ Trees/acre } & \multirow[b]{2}{*}{$\mathrm{BA} / \mathrm{acre}$} & \multirow[b]{2}{*}{$\mathrm{CCF}$} & \multicolumn{3}{|c|}{ Volume per acre } & \multirow[b]{2}{*}{ Accretion } & \multirow[b]{2}{*}{ Mortality } & \multirow[b]{2}{*}{$\bar{x}$ d.b.h. } & \multirow[b]{2}{*}{$\bar{x}$ height } \\
\hline & & & & $\begin{array}{c}\text { Total } \\
\mathrm{ft}^{3} \\
\end{array}$ & $\begin{array}{c}\text { Merchantable } \\
\mathrm{ft}^{3}\end{array}$ & $\begin{array}{l}\text { Scribner } \\
\text { bd.ft. }\end{array}$ & & & & \\
\hline & & $F t^{2}$ & & & & & $F t^{3}$ & $F t^{3}$ & Inches & Feet \\
\hline 45 & 55 & 33 & 44 & 719 & 633 & 2,043 & 79 & 4 & 10.5 & 55.5 \\
\hline 55 & 52 & 54 & 61 & 1,475 & 1,328 & 6,068 & 90 & 7 & 13.7 & 73.5 \\
\hline 65 & 50 & 72 & 75 & 2,299 & 2,105 & 10,492 & 94 & 11 & 16.4 & 86.2 \\
\hline 75 & 47 & 89 & 85 & 3,124 & 2,888 & 14,902 & 107 & 16 & 18.6 & 96.3 \\
\hline 85 & 45 & 106 & 96 & 4,037 & 3,740 & 19,992 & 110 & 20 & 20.8 & 104.9 \\
\hline 95 & 42 & 121 & 104 & 4,928 & 4,577 & 25,063 & 119 & 26 & 22.9 & 112.4 \\
\hline 105 & 40 & 137 & 112 & 5,864 & 5,452 & 30,514 & 123 & 31 & 25.1 & 119.0 \\
\hline 115 & 38 & 153 & 120 & 6,779 & 6,313 & 36,202 & 119 & 37 & 27.2 & 125.0 \\
\hline 125 & 36 & 166 & 126 & 7,596 & 7,090 & 41,353 & 123 & 43 & 29.2 & 130.4 \\
\hline 135 & 33 & 178 & 131 & 8,396 & 7,855 & 46,442 & 118 & 48 & 31.3 & 135.3 \\
\hline 145 & 31 & 187 & 134 & 9,091 & 8,530 & 51,063 & 0 & 0 & 33.1 & 139.8 \\
\hline
\end{tabular}


Table 38.--Yield table for Stand 34, Colville National Forest, Sullivan Lake Ranger District, Hanlon Meadows

\begin{tabular}{|c|c|c|c|c|c|c|c|c|c|c|}
\hline \multirow[b]{2}{*}{ Age } & \multirow[b]{2}{*}{ Trees/acre } & \multirow[b]{2}{*}{$\mathrm{BA} /$ acre } & \multirow[b]{2}{*}{$\mathrm{CCF}$} & \multicolumn{3}{|c|}{ Volume per acre } & \multirow[b]{2}{*}{ Accretion } & \multirow[b]{2}{*}{ Mortality } & \multirow[b]{2}{*}{$\bar{x} \mathrm{~d}, \mathrm{~b}, \mathrm{~h}$. } & \multirow[b]{2}{*}{$\bar{x}$ height } \\
\hline & & & & $\begin{array}{c}\text { Total } \\
\mathrm{ft}^{3}\end{array}$ & $\begin{array}{c}\text { Merchantable } \\
\mathrm{ft}^{3}\end{array}$ & $\begin{array}{c}\text { Scribner } \\
\text { bd.ft. }\end{array}$ & & & & \\
\hline & & $F t^{2}$ & & & & & $F t^{3}$ & $F t^{3}$ & Inches & Feet \\
\hline 45 & 67 & 28 & 39 & 837 & 664 & 1,767 & 48 & 2 & 8.8 & 61.6 \\
\hline 55 & 65 & 39 & 50 & 1,296 & 1,006 & 3,511 & 53 & 3 & 10.4 & 69.8 \\
\hline 65 & 64 & 49 & 59 & 1,793 & 1,356 & 5,299 & 53 & 6 & 11.9 & 77.2 \\
\hline 75 & 62 & 57 & 66 & 2,266 & 1,662 & 6,746 & 58 & 10 & 13.1 & 84.0 \\
\hline 85 & 59 & 65 & 72 & 2,746 & 1,955 & 7,927 & 61 & 16 & 14.2 & 90.4 \\
\hline 95 & 56 & 71 & 76 & 3,195 & 2,206 & 8,788 & 61 & 27 & 15.3 & 96.4 \\
\hline 105 & 52 & 75 & 78 & 3,537 & 2,370 & 9,248 & 58 & 40 & 16.3 & 102.1 \\
\hline 115 & 47 & 75 & 77 & 3,720 & 2,429 & 9,299 & 58 & 49 & 17.1 & 107.5 \\
\hline 125 & 42 & 74 & 75 & 3,804 & 2,429 & 9,136 & 54 & 54 & 18.0 & 112.5 \\
\hline 135 & 37 & 72 & 72 & 3,798 & 2,379 & 8,795 & 51 & 54 & 18.9 & 117.3 \\
\hline 145 & 32 & 69 & 69 & 3,763 & 2,304 & 8,338 & 0 & 0 & 19.8 & 121.9 \\
\hline
\end{tabular}

Table 39.--Yield table for Stand 35, Colville National Forest Colville Ranger District, Joslin Meadow

\begin{tabular}{|c|c|c|c|c|c|c|c|c|c|c|}
\hline \multirow[b]{2}{*}{ Age } & \multirow[b]{2}{*}{ Trees/acre } & \multirow[b]{2}{*}{ BA/acre } & \multirow[b]{2}{*}{$\mathrm{CCF}$} & \multicolumn{3}{|c|}{ Volume per acre } & \multirow[b]{2}{*}{ Accretion } & \multirow[b]{2}{*}{ Mortality } & \multirow[b]{2}{*}{$\bar{x}$ d.b.h. } & \multirow[b]{2}{*}{$\bar{x}$ height } \\
\hline & & & & $\begin{array}{c}\text { Total } \\
\mathrm{ft}^{3} \\
\end{array}$ & $\begin{array}{c}\text { Merchantable } \\
\mathrm{ft}^{3}\end{array}$ & $\begin{array}{l}\text { Scribner } \\
\text { bd.ft. }\end{array}$ & & & & \\
\hline & & $F t^{2}$ & & & & & $F t^{3}$ & $F t^{3}$ & Inches & Feet \\
\hline 54 & 141 & 57 & 72 & 2,028 & 1,655 & 6,244 & 118 & 14 & 8.6 & 69.4 \\
\hline 64 & 131 & 78 & 91 & 3,070 & 2,643 & 11,436 & 103 & 21 & 10.5 & 80.5 \\
\hline 74 & 122 & 93 & 102 & 3,884 & 3,350 & 16,125 & 103 & 31 & 11.8 & 86.5 \\
\hline 84 & 114 & 105 & 109 & 4,599 & 3,940 & 19,740 & 113 & 47 & 13.0 & 92.1 \\
\hline 94 & 104 & 114 & 114 & 5,257 & 4,490 & 22,963 & 112 & 72 & 14.1 & 97.5 \\
\hline 104 & 94 & 117 & 115 & 5,656 & 4,812 & 24,778 & 108 & 101 & 15.1 & 102.6 \\
\hline 114 & 82 & 114 & 111 & 5,720 & 4,849 & 25,116 & 95 & 126 & 16.0 & 107.5 \\
\hline 124 & 69 & 105 & 103 & 5,409 & 4,594 & 23,904 & 86 & 127 & 16.7 & 112.2 \\
\hline 134 & 58 & 95 & 94 & 5,003 & 4,266 & 22,238 & 71 & 119 & 17.4 & 116.9 \\
\hline 144 & 47 & 84 & 84 & 4,521 & 3,886 & 20,395 & 58 & 111 & 18.1 & 121.7 \\
\hline 154 & 38 & 73 & 75 & 3,990 & 3,446 & 18,082 & 0 & 0 & 18.8 & 126.6 \\
\hline
\end{tabular}

Table 40.--Yield table for Stand 36, Colville National Forest, Sullivan Lake Ranger District, Johns Creek

\begin{tabular}{|c|c|c|c|c|c|c|c|c|c|c|}
\hline \multirow[b]{2}{*}{ Age } & \multirow[b]{2}{*}{ Trees/acre } & \multirow[b]{2}{*}{$\mathrm{BA} / \mathrm{acre}$} & \multicolumn{4}{|c|}{ Volume per acre } & \multirow[b]{2}{*}{ Accretion } & \multirow[b]{2}{*}{ Mortality } & \multirow[b]{2}{*}{$\bar{x}$ d.b.h. } & \multirow[b]{2}{*}{$\bar{x}$ height } \\
\hline & & & CCF & $\begin{array}{l}\text { Total } \\
\mathrm{ft}^{3}\end{array}$ & $\begin{array}{c}\text { Merchantable } \\
\mathrm{ft}^{3}\end{array}$ & $\begin{array}{l}\text { Scribner } \\
\text { bd.ft. }\end{array}$ & & & & \\
\hline & & $F t^{2}$ & & & & & $F t^{3}$ & $F t^{3}$ & Inches & Feet \\
\hline 15 & 173 & 11 & 25 & 104 & 8 & 0 & 22 & 2 & 3.4 & 20.1 \\
\hline 25 & 145 & 21 & 38 & 305 & 140 & 22 & 44 & 4 & 5.1 & 34.1 \\
\hline 35 & 128 & 35 & 54 & 710 & 720 & 752 & 44 & 6 & 7.1 & 49.1 \\
\hline 45 & 116 & 45 & 63 & 1,084 & 1,184 & 2,088 & 49 & 8 & 8.4 & 60.4 \\
\hline 55 & 108 & 54 & 71 & 1,501 & 1,625 & 4,549 & 63 & 9 & 9.6 & 71.0 \\
\hline 65 & 101 & 65 & 79 & 2,035 & 1,952 & 7,611 & 72 & 12 & 10.9 & 80.8 \\
\hline 75 & 95 & 77 & 88 & 2,634 & 2,362 & 10,873 & 75 & 16 & 12.1 & 89.9 \\
\hline 85 & 90 & 87 & 94 & 3,224 & 2,877 & 14,131 & 80 & 23 & 13.3 & 98.3 \\
\hline 95 & 84 & 96 & 100 & 3,803 & 3,419 & 17,410 & 80 & 31 & 14.5 & 106.2 \\
\hline 105 & 78 & 102 & 104 & 4,295 & 3,880 & 20,196 & 80 & 45 & 15.5 & 113.5 \\
\hline 115 & 72 & 106 & 105 & 4,639 & 4,234 & 22,324 & 0 & 0 & 16.4 & 120.6 \\
\hline
\end{tabular}


Graham, R. T., and J. R. Tonn.

1980. Case study: growth and development of forest stands in the Northern Rocky Mountains. USDA For. Serv. Res. Pap.

INT-255, 24 p. Intermt. For. and Range Exp. Stn., Ogden, Utah 84401 .

Tree diameter and height growth were compared for nine habitat types. Ten-year diameter growth was highly variable among habitat types as was 10-year height growth. High variation in both diameter and height growth also occurred within habitat types. Thirty-six local yield tables are presented, one for each stand in the study.

KEYWORDS: Stand yield, diameter growth, height growth, habitat type, Northern Rocky Mountains

Graham, R. T., and J. k. Tonn.

1980. Case study: growth and development of forest stands in the Northern Rocky Mountains. USDA For. Serv. Res. Pap. INT-255, 24 p. Intermt. For. and Range Exp. Stn., Ogden, Utah 84401 .

Tree diameter and height growth were compared for nine habitat types. Ten-year diameter growth was highly variable among habitat types as was 10-year height growth. High variation in both diameter and height growth also occurred within habitat types. Thirty-six local yield tables are presented, one for each stand in the study.

KEYWORDS: Stand yield, diameter growth, height growth, habitat type, Northern Rocky Mountains 
The Intermountain Station, headquartered in Ogden, Utah, is one of eight regional experiment stations charged with providing scientific knowledge to help resource managers meet human needs and protect forest and range ecosystems.

The Intermountain Station includes the States of Montana, Idaho, Utah, Nevada, and western Wyoming. About 231 million acres, or 85 percent, of the land area in the Station territory are classified as forest and rangeland. These lands include grasslands, deserts, shrublands, alpine'areas, and well-stocked forests. They supply fiber for forest industries; minerals for energy and industrial development; and water for domestic and industrial consumption. They also provide recreation opportunities for millions of visitors each year.

Field programs and research work units of the Station are maintained in:

Boise, Idaho

Bozeman, Montana (in cooperation with Montana State University)

Logan, Utah (in cooperation with Utah State University)

Missoula, Montana (in cooperation with the University of Montana)

Moscow, Idaho (in cooperation with the University of Idaho)

Provo, Utah (in cooperation with Brigham Young University)

Reno, Nevada (in cooperation with the University of Nevada)

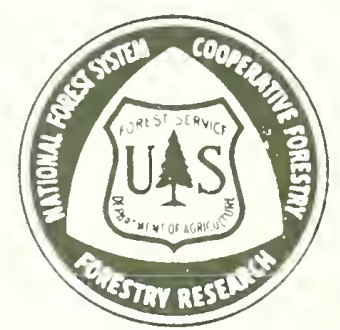

